\title{
Spatial positioning and matrix programs of cancer-associated fibroblasts promote T cell exclusion in human lung tumors
}

John A. Grout ${ }^{1,2,3}$, Philémon Sirven ${ }^{4,5}$, Andrew M. Leader ${ }^{1,2,3}$, Shrisha Maskey ${ }^{1,2,3}$, Eglantine Hector ${ }^{4,5}$, Isabelle Puisieux ${ }^{4,5}$, Fiona Steffan ${ }^{4,5}$, Evan Cheng ${ }^{1,2,3}$, Navpreet Tung ${ }^{1,2,3}$, Mathieu Maurin ${ }^{4,5}$, Romain Vaineau $^{4,5}$, Léa Karpf ${ }^{1,2,3}$, Martin Plaud ${ }^{1,2,3}$, Maria Casanova-Acebes ${ }^{1,2,3}$, Alexandra Tabachnikova ${ }^{1,2,3}$, Shilpa Keerthivasan ${ }^{6}$, Alona Lansky ${ }^{1,2,3}$, Jessica LeBérichel ${ }^{1,2,3}$, Laura Walker ${ }^{1,7}$, Adeeb H. Rahman ${ }^{1,7,8}$, Sacha Gnjatic ${ }^{1,2,7,9}$, Julien Adam ${ }^{10}$, Jerome C. Martin ${ }^{1,2,3}$, Andrea Wolf ${ }^{11}$, Raja Flores ${ }^{11}$, Mary Beth Beasley $^{12}$, Rachana Pradhan ${ }^{13}$, Sören Müller ${ }^{13}$, Thomas U. Marron ${ }^{1,2,9}$, Shannon J. Turley ${ }^{6}$, Miriam Merad $^{1,2,3,7}$, Ephraim Kenigsberg ${ }^{1,8,14}$, Hélène Salmon ${ }^{1,2,3,4,5,14}$

${ }^{1}$ The Precision Immunology Institute, Icahn School of Medicine at Mount Sinai, New York, NY, USA.

${ }^{2}$ The Tisch Cancer Institute, Icahn School of Medicine at Mount Sinai, New York, NY, USA.

${ }^{3}$ Department of Oncological Sciences, Icahn School of Medicine at Mount Sinai, New York, NY, USA.

${ }^{4}$ Institut Curie, INSERM, U932, Equipe Leader Fondation ARC 2018, Paris, France.

${ }^{5}$ PSL Research University, Paris, France.

${ }^{6}$ Department of Cancer Immunology, Genentech, Inc., South San Francisco, CA, USA.

${ }^{7}$ Human Immune Monitoring Center, Icahn School of Medicine at Mount Sinai, New York, NY, USA.

${ }^{8}$ Department of Genetics and Genomic Sciences, Icahn School of Medicine at Mount Sinai, New York, NY, USA.

${ }^{9}$ Division of Hematology/Oncology, Icahn School of Medicine at Mount Sinai, New York, NY, USA

${ }^{10}$ Department of Pathology, Paris Saint-Joseph Hospital, Paris, France

${ }^{11}$ Department of Thoracic Surgery, Icahn School of Medicine at Mount Sinai, New York, NY, USA.

${ }^{12}$ Department of Pathology, Icahn School of Medicine at Mount Sinai, New York, NY, USA.

${ }^{13}$ Department of Bioinformatics and Computational Biology, Genentech, South San Francisco, CA, USA

${ }^{14}$ Senior authors

*Correspondence: helene.salmon@curie.fr, ephraim.kenigsberg@mssm.edu 


\section{SUMMARY}

2 It is currently accepted that activated cancer-associated fibroblasts (CAF) participate in $\mathrm{T}$ cell

3 exclusion from tumor nests, but it remains unclear how they promote barrier phenotypes, and

4 whether specific subsets are involved. Here, using single-cell RNA sequencing coupled with

5 multiplex imaging on a large cohort of lung tumors, we identify four main CAF populations, of

6 which only two are associated with T cell exclusion: (i) $\mathrm{MYH}_{1} 1^{+} \alpha \mathrm{SMA}^{+} \mathrm{CAF}$, which are present

7 in early-stage tumors and form a single-cell layer lining cancer aggregates, and (ii) $\mathrm{FAP}^{+} \alpha \mathrm{SMA}^{+}$

8 CAF, which appear in more advanced tumors and organize in patches within the stroma or in

9 multiple layers around tumor nests. Both CAF populations show a contractility phenotype together

10 with dense and aligned matrix fiber deposition compared to the T cell-permissive CAF. Yet they

11 express distinct matrix genes, including COL4A1/COL9A1 (MYH1 $\left.{ }^{+} \alpha \mathrm{SMA}^{+} \mathrm{CAF}\right)$ and

$12 \mathrm{COL11A} 1 / \mathrm{COL} 12 \mathrm{~A} 1\left(\mathrm{FAP}^{+} \alpha \mathrm{SMA}^{+} \mathrm{CAF}\right)$. Hereby, we uncovered unique molecular programs of

13 CAF driving $\mathrm{T}$ cell marginalization, whose targeting should increase immunotherapy efficacy in 14 patients bearing $\mathrm{T}$ cell-excluded tumors.

\section{SIGNIFICANCE}

17 The cellular and molecular programs driving $\mathrm{T}$ cell marginalization in solid tumors remain unclear.

18 Here, we describe two CAF populations associated with T cell exclusion in human lung tumors.

19 We demonstrate the importance of pairing molecular and spatial analysis of the tumor 20 microenvironment, a prerequisite to develop new strategies targeting $\mathrm{T}$ cell-excluding CAF. 


\section{INTRODUCTION}

22 Lung cancer is the leading cause of cancer-related deaths worldwide, accounting for roughly 1.6

23 million deaths per year, with non-small cell lung carcinoma (NSCLC) being the most prevalent

24 form (1). The partial success of immune checkpoint blockade in only a subset of NSCLC patients

25 underscores the need for a better understanding of the determinants controlling anti-tumor

26 immunity (2). In addition to high tumor mutational burden and PD-L1 expression levels in the

27 tumor, $\mathrm{CD}^{+} \mathrm{T}$ cell density has been shown as a predictor of immunotherapy response $(3,4)$. By

28 analyzing the $\mathrm{T}$ cell localization within the tumor, recent studies have revealed the importance of

29 T cell infiltration into the tumor nests relative to the surrounding stroma $(3,5,6)$. Understanding

30 the mechanisms regulating $\mathrm{T}$ cell exclusion are therefore crucial to improve $\mathrm{T}$ cell-based therapies

31 and patient outcomes.

33 Using real-time imaging of T cell dynamics in human NSCLC, we previously found that dense

34 fibers oriented parallel to the tumor-stroma interface form a barrier around the tumor mass and

35 limit $\mathrm{T}$ cell contact with tumor cells (7). However, the cellular sources and their extracellular

36 matrix (ECM) programs remain unknown. Fibroblasts are known to shape lymphocyte

37 compartmentalization in secondary lymphoid organs, where they produce distinct sets of

38 chemokines and a complex ECM conduit system that serves as a scaffold along which dendritic

39 cells and lymphocytes migrate and engage (8-10). While the role of fibroblasts in restricting

40 immune cell localization is well established in spleen and lymph nodes, only recently has the tumor

41 stroma emerged as a player in regulating local immune responses (11-14).

42 
43 Given the growing evidence indicating that cancer-associated fibroblasts (CAF) can regulate tumor

44 immunity and progression(11-14), CAF are becoming an important target for cancer treatment.

45 TGF $\beta$ blockade and NOX4 inhibition were shown to act on CAF and facilitate T cell infiltration,

46 leading to better responses to anti-PD-1/PD-L1 treatment in murine cancer models $(6,15,16)$. Yet

47 modulating and depleting CAF have led to opposite results in other tumor systems $(16,17)$ and has

48 not yet managed to achieve clinical benefit in human cancer $(18,19)$. How to manipulate fibroblast

49 properties for therapeutic purpose remains challenging, largely due to our limited understanding

50 of the tumor CAF compartment and the mechanisms by which distinct CAF populations modulate

51 anti-tumor immunity, including immune cell spatial organization.

53 The initial characterization of functional heterogeneity of CAF included description of

54 inflammatory CAF (iCAF) and myofibroblastic CAF (myCAF) in mouse models of pancreatic

55 cancer(20). Transcriptional signatures of these distinct CAF phenotypes have subsequently been

56 found in human pancreatic and breast cancer(21,22), as well as an additional subset, antigen-

57 presenting CAF (apCAF)(22). iCAF are described as being found distal from the tumor site with

58 a secretory phenotype whereas myCAF are characterized by activation and contractility genes and

59 their close proximity to tumor cells(20). Prior studies have used single-cell RNA sequencing

60 (scRNAseq) to profile CAF in various human cancers, including NSCLC(23), bladder(24),

61 pancreas(22,25), breast(21), head and neck(26), and liver(27). While the diversity of CAF is

62 increasingly appreciated, the molecular programs of human fibroblast subsets and their discrete

63 functional contributions to the tumor organization and T cell compartmentalization have not been

64 resolved. 
66 We reasoned that pairing scRNAseq profiling with high resolution spatial mapping would enable

67 unbiased identification of CAF transcriptional subsets and uncovering their spatial organization in

68 the context of the tumor microenvironment. Our scRNAseq analysis on 15 surgically resected

69 NSCLC samples along with 12 paired adjacent tissue samples identified novel CAF

70 subpopulations which we validated by profiling 35 tumors by multiplexed immunohistochemistry

71 (IHC) (28). We analyzed the spatial organization of the stromal and immune cell populations, and

72 revealed two CAF subsets with distinct ECM programs that were associated with $\mathrm{CD}^{+}$and $\mathrm{CD}^{+}$

$73 \mathrm{~T}$ cell exclusion from the tumor nests. Importantly, by applying high-resolution histological

74 profiling on a large NSCLC cohort, our study characterizes both the intra-tumor and inter-tumor

75 CAF and $\mathrm{T}$ cell heterogeneity.

\section{RESULTS}

78 Paired scRNAseq and IHC analysis identifies four CAF populations with distinct transcriptional profiles and structural organization in human NSCLC

81 To characterize the stromal cell compartment in NSCLC in an unbiased way, we profiled non-

82 immune, non-tumor/epithelial cells isolated from 15 NSCLC samples and 12 paired adjacent tissue

83 samples using the 10x Genomics scRNAseq platform (Figure 1A, Table 1). Using flow cytometry

84 and mass cytometry by Time of Flight (CyTOF), we optimized the digestion and sorting protocols

85 to maximize the stromal cell recovery while preserving cell integrity (Methods, Figures S1A,

86 S1B). 33,742 cells were sequenced in total which contained 31,402 stromal cells after excluding

87 contaminating immune cells, epithelial cells, and cells not passing quality control (Table 2). Using

88 an unsupervised clustering, which integrates samples over different conditions and patients while 
A

15 NSCLC

\& 12 paired adjacent lung samples

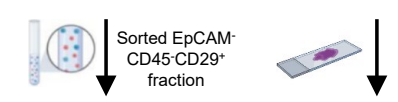

Single cell RNA-seq ( $n=31,402$ stromal cells)
35 NSCLC

FFPE samples

Multiplex imaging

\section{B}

Smooth muscle cells

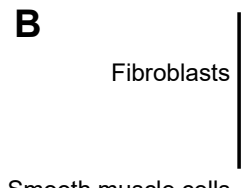

Pericytes

Blood endothelial cells (EC)

Lymphatic EC

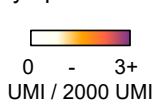

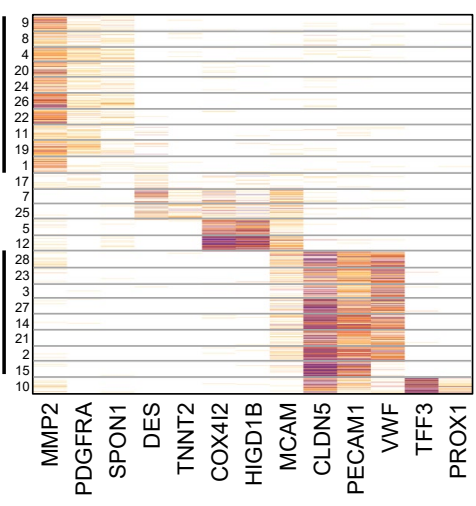

D Tissue enrichment

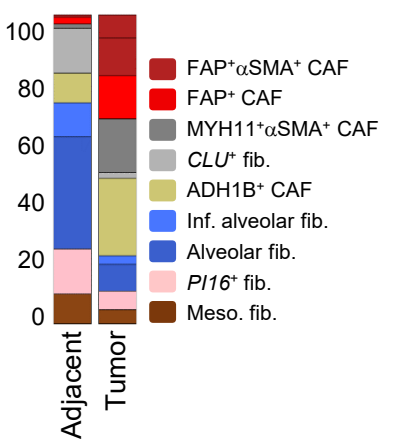

C

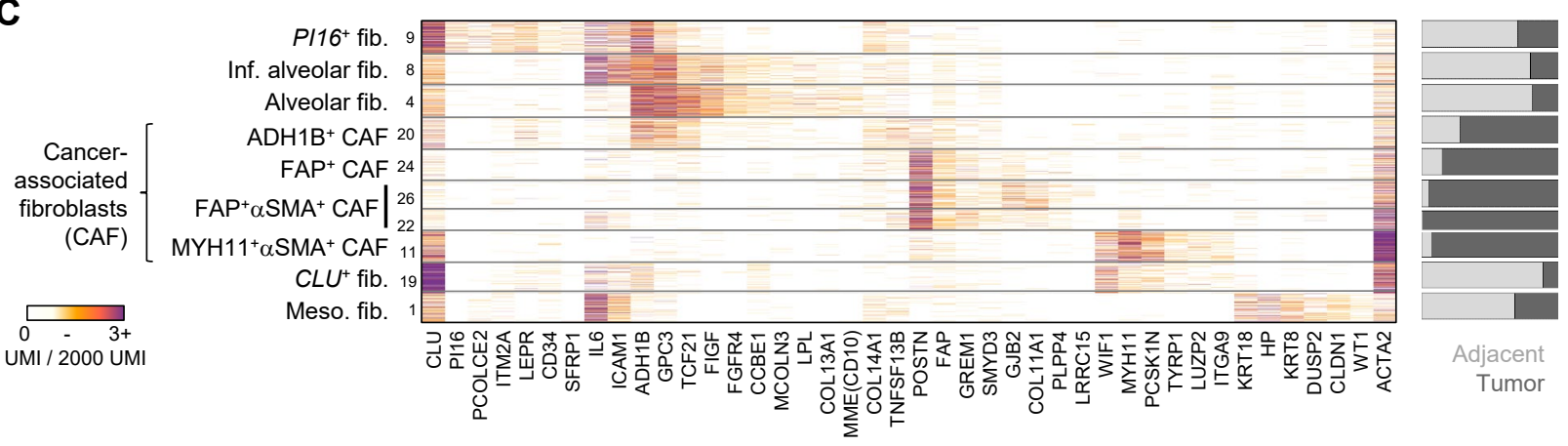

E $\quad P / 16^{+}$fib.
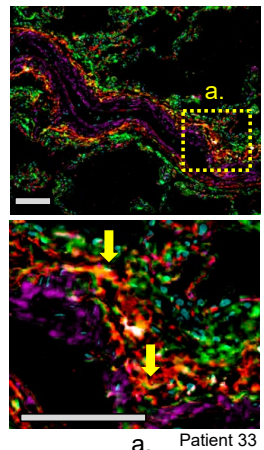

Nuclei Keratin ADH1B

CD34 MYH11
Alveolar fib.

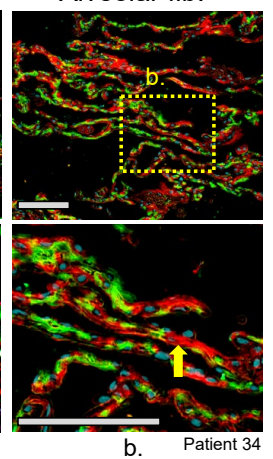

Nuclei CD10

CD34
$\mathrm{ADH}_{1 \mathrm{~B}^{+}} \mathrm{CAF}$

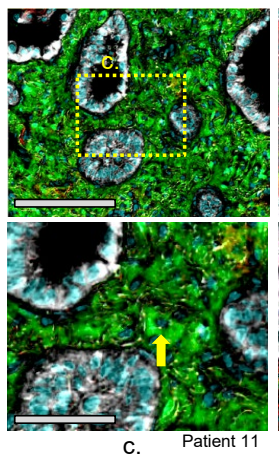

c.

Nuclei Keratin

CD10 ADH1B
$\mathrm{FAP}^{+} \mathrm{CAF}$

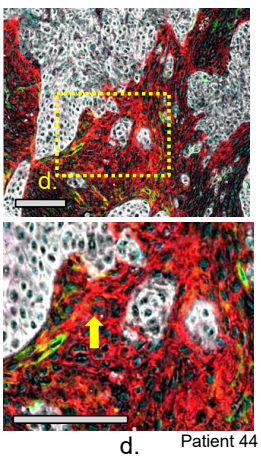

Nuclei Keratin.

aSMA FAP ADH1B

$\mathrm{FAP}^{+} \alpha \mathrm{SMA}^{+} \mathrm{CAF}$

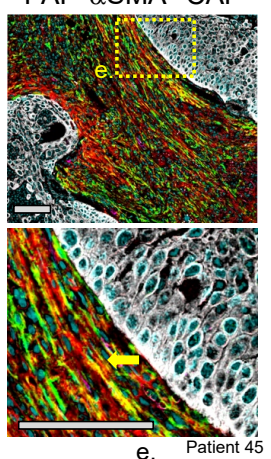

Nuclei Keratin

aSMA FAP CD34

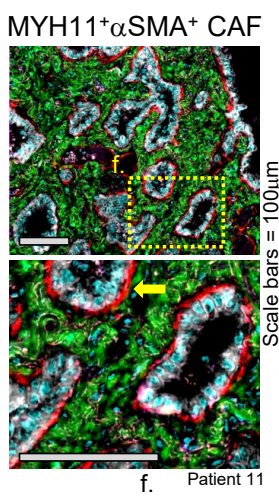

Nuclei Keratin FAP

ADH1B MYH11

F

IHC staining of fibroblasts and CAF

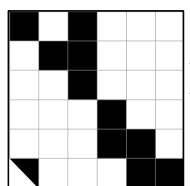

Pl16 fib.

Alveolar fib.

$\mathrm{ADH}^{\mathrm{B}} \mathrm{B}^{+} \mathrm{CAF}$

$\mathrm{FAP}^{+} \mathrm{CAF}$

$\mathrm{FAP}^{+} \alpha \mathrm{SMA}^{+} \mathrm{CAF}$

$\mathrm{MYH}^{1} 11^{+} \alpha \mathrm{SMA}{ }^{+} \mathrm{CAF}$

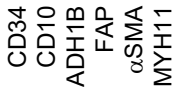

Negative

Positive 
Fig. 1 | Paired scRNAseq and IHC analysis identifies four CAF populations with distinct transcriptional profiles and structural organization in human NSCLC. A, Tissue processing workflow for scRNAseq and IHC imaging of FFPE samples. B, scRNAseq mRNA counts (unique molecular identifiers, UMI) per cell (rows) of select stromal lineage marker genes (columns). Fibroblast, smooth muscle, pericyte, blood and lymphatic endothelial cell clusters are identified based on expression of marker genes such as, PDGFRA, DES, COX4I2, PECAM1, and TFF3, respectively. All cells displayed in this figure, and all subsequent similar scRNAseq figures, were downsampled to 2000 UMI. C, Extended gene lists highlighting gene expression profiles between the fibroblast subsets along with differing propensities for enrichment (right bar plot) in tumor (dark gray) or adjacent tissue (light gray). D, Averaged fibroblast composition in adjacent and tumor samples across all patients. The bar graph depicts the percentage of cells from each fibroblast subset among all fibroblasts. E, FFPE NSCLC sections were stained for fibroblast markers identified in scRNAseq results. All the scRNAseq-based fibroblast clusters (D) were detected utilizing IHC except meso. fib. and $\mathrm{CLU}^{+}$fib., which were not in the scope of this study. Arrows highlight cells of interest $\left(P I 16^{+}\right.$fib.: $\mathrm{CD}^{+} 4^{+} \mathrm{ADH} 1 \mathrm{~B}^{+} \mathrm{MYH1} 1^{\text {neg }}$, Alv. fib.: $\mathrm{CD} 10^{+} \mathrm{CD} 34^{\text {neg }}, \mathrm{ADH}_{1 B^{+}} \mathrm{CAF}: \mathrm{ADH}^{+} \mathrm{CD} 10^{\text {neg }}, \mathrm{FAP}^{+} \mathrm{CAF}: \mathrm{FAP}^{+} \mathrm{ADH}^{\mathrm{neg}} \mathrm{\alpha SMA}^{\text {neg }}$; $\mathrm{FAP}^{+} \alpha \mathrm{SMA}^{+} \mathrm{CAF}: \mathrm{FAP}^{+} \alpha \mathrm{SMA}^{+} \mathrm{CD} 34^{\text {neg }}$; MYH1 $\left.{ }^{+} \alpha \mathrm{SMA}^{+} \mathrm{CAF}: \mathrm{MYH} 11^{+} \mathrm{FAP}^{\text {neg }} \mathrm{ADH} 1 \mathrm{~B}^{\text {neg }}\right)$. See Figure S3 for other stainings. All scale bars are $100 \mu \mathrm{m}$. F, IHC staining presentation for the main identified fibroblast and CAF clusters. 
modeling background noise $(29,30)$, we identified 28 clusters, including; 24 stromal cell clusters

90 of variable abundance shared among samples (Figure S1C, table 2) as well as 4 clusters, either

91 containing contaminating immune cells or cells with high mitochondrial content, that were

92 excluded from future analysis. mRNA counts (unique molecular identifiers, UMI) per cluster and

93 mitochondrial content per sample were similar (Figures S1C, S1D).

95 To unbiasedly dissect cell identities, we analyzed the mRNA counts of variably expressed genes

96 across the 24 stromal cell clusters (Figure S1E). The cell clusters represented 3 major stromal cell

97 compartments and expressed well reported lineage markers: fibroblasts (PDGFRA ${ }^{+}, \mathrm{MMP}^{+}$),

98 endothelial cells [EC, including both blood $\left(\mathrm{CLDN}^{+}, \mathrm{PECAM}^{+}\right)$and lymphatic $\left(\mathrm{TFF}^{+}\right.$,

$\left.\left.99 \mathrm{PROX}^{+}\right) \mathrm{EC}\right]$, and perivascular cells $\left[\mathrm{PvC}\right.$, including pericytes $\left(\mathrm{MCAM}^{+}, \mathrm{COX} 4 \mathrm{I}^{+}\right)$, and smooth

100 muscle $(\mathrm{SM})$ cells $\left(\mathrm{MCAM}^{+}, \mathrm{DES}^{+}\right)$] (Figures 1B, S1F). Blood EC clusters included arteries,

101 venules, tip cells, as well as two lung capillary subsets recently described as aerocytes and general

102 capillaries(31) (Figure S2A). The PvC clusters enriched in tumor lesions included tumor pericytes,

103 which expressed high amounts of RGS5 and multiple collagens (COL1A1, COL3A1, COL6A3),

104 and a cluster expressing multiple immunomodulatory genes including CCL19 and CCL21 (Figure

105 S2B). To be noted, IHC showed that the $\mathrm{MCAM}^{+}$cells were restricted to vascular areas and were

106 not found in the rest of the stroma (Figure S2C).

107

108 Further dissection of fibroblast populations identified multiple subsets with distinct transcriptional

109 profiles and uneven abundances in the tumor lesion or the adjacent tissue (Figures 1C, 1D, Table

110 3). Based on this scRNAseq analysis, we identified genes associated with each cluster and defined

111 antibody panels (Table 4) that enabled further characterization by multiplexed IHC (Figures 1E, 
1F, S3A-B). Two clusters enriched in the adjacent lung tissue were characterized by co-expression

113 of $M M E$ (CD10), FIGF (VEGFD), FGFR4 (Figure 1C) and were annotated as alveolar fibroblasts

114 (alv. fib.) based on their specific localization to the lung alveoli by IHC (Figures 1E, S3A).

115 Interestingly, one of these clusters expressed high levels of inflammatory genes, including IL6 and

116 ICAM1, and was thus referred to as inflamed alveolar fibroblasts (inf. alv. fib.) (Figure 1C).

117 Another cluster enriched in the adjacent lung was annotated as $\mathrm{PIIG}^{+}$fibroblasts based on its co-

118 expression of PI16, CD34 and LEPR (leptin receptor), localization to the blood vessel adventitia,

119 and similarity to the universal PII6 $^{+}$fibroblasts described in Buechler et al., 2021 (Figures 1C, 1E,

120 S3A) (32-34). The last adjacent tissue cluster, $\mathrm{CLU}^{+}$fib., was characterized by high expression of

$121 C L U$ (clusterin) (Figure 1C).

123 Fibroblast clusters enriched in tumor samples were annotated as CAF. One CAF cluster displayed

124 an expression profile similar to that of alv. fib., including expression of the broad adjacent tissue

125 fibroblast marker, $A D H 1 B$ (alcohol dehydrogenase 1B), and lower expression of the canonical

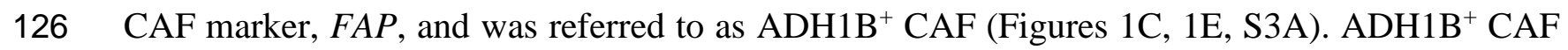

127 could be distinguished from alv. fib. in IHC by their lack of CD10 expression and localization in

128 the tumor lesion (Figures 1C, 1E, S3A, S3C). Three clusters showed strong expression of canonical

129 activated CAF markers, FAP, POSTN, LRRC15 and GREM1 $(23,25)$ and were denoted as FAP ${ }^{+}$

130 CAF (Figures 1C, 1E, S3B). Another common CAF marker, ACTA2 ( $\alpha \mathrm{SMA}$ ) (35), was

131 differentially expressed among the FAP ${ }^{+}$CAF (Figures 1C, 1E, S3B) and clusters with high

132 ACTA2 expression were designated as $\mathrm{FAP}^{+} \alpha \mathrm{SMA}^{+} \mathrm{CAF}$. Notably, a cluster that was highly

133 enriched in a single patient (Table 2) shared both fibroblast genes (PDGFRA, MMP2, COL1A1,

$134 B G N$ ) and mesothelial cell genes, such as keratins and WT1, and was therefore designated as 
mesothelial-like fibroblasts (meso. fib.) (36) (Figure 1C). An additional CAF cluster, $\mathrm{MYH} 11^{+} \alpha \mathrm{SMA}^{+} \mathrm{CAF}$, clearly distinct from the other CAF subsets, was characterized by the expression of MYH11 (myosin heavy chain 11), ACTA2 and intermediate levels of CD34, while lacking $A D H 1 B$ and $F A P$ expression (Figure 1C). Histological analysis of matched formalin-fixed paraffin-embedded (FFPE) tumor samples revealed a $\mathrm{MYH} 11^{+} \alpha \mathrm{SMA}^{+} \mathrm{CD} 34^{+} \mathrm{ADH} 1 \mathrm{~B}^{\text {neg }} \mathrm{FAP}^{\text {neg }}$ cell population observed as a single layer of elongated CAF encapsulating tumor nests, in contrast to $\mathrm{ADH}_{1 \mathrm{~B}}^{+} \mathrm{CAF}$ and $\mathrm{FAP}^{+} \mathrm{CAF}$ that are spread throughout the stroma (Figures $\left.1 \mathrm{E}, 1 \mathrm{~F}, \mathrm{~S} 3 \mathrm{~A}\right)$. CyTOF confirmed the presence of the main fibroblast subsets identified through scRNAseq, including alv. fib., $\mathrm{PI}^{+}$fib., $\mathrm{MYH} 11^{+} \alpha \mathrm{SMA}^{+} \mathrm{CAF}$ and $\mathrm{FAP}^{+} \alpha \mathrm{SMA}^{+} \mathrm{CAF}$ (Figure $2 \mathrm{~A}$ ).

145 Further analysis of $\mathrm{ADH}_{1 \mathrm{~B}}{ }^{+} \mathrm{CAF}$ revealed a subset of cells that expressed high levels of $\mathrm{T}$ cell146 attracting and T cell-retention genes CCL19, CCL21, and VCAM1, reminiscent of fibroblastic 147 reticular cells present in secondary lymphoid organs (37) (Figure 2B). IHC staining of CCL19

148 confirmed the specific localization of these fibroblasts to tertiary lymphoid structures, with clear 149 preferential enrichment for the $\mathrm{T}$ cell zone (Figure 2B). In some cases, the B cell zone was 150 delineated by podoplanin expression, which marks follicular dendritic cells that were not captured by scRNAseq, likely due to their low abundance (Figure $2 \mathrm{~B}$ ).

153 Taken together, our combined IHC and single-cell analysis has defined diverse fibroblast

154 populations with distinct molecular and spatial patterns in human NSCLC. By enriching for 155 stromal cells from a large NSCLC cohort, we achieved highly granular scRNAseq characterization 156 and uncovered CAF populations undescribed to date, including a single layer of $\mathrm{MYH}_{1}{ }^{+} \alpha \mathrm{SMA}^{+}$ 157 CAF bordering tumor cells in a fraction of NSCLC lesions. The four CAF subsets described here 
A

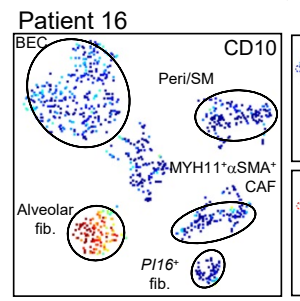

CyTOF - viSNE

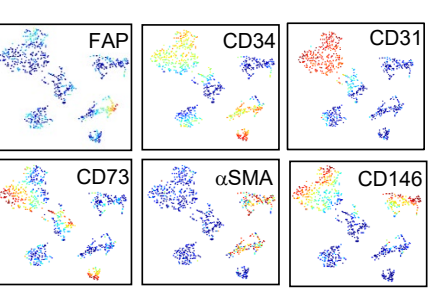

Patient 17

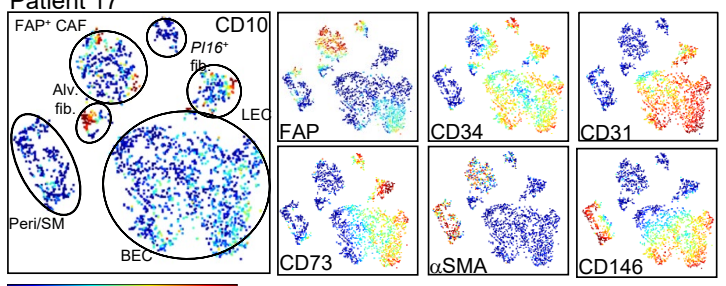

Staining intensity
B

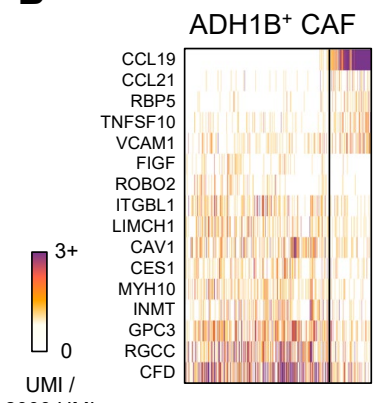
2000 UMI
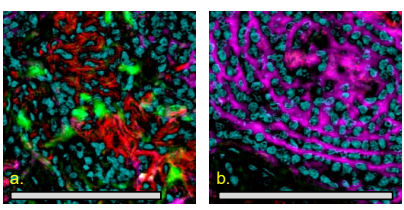

CCL19+ cells

in tertiary lymphoid structures
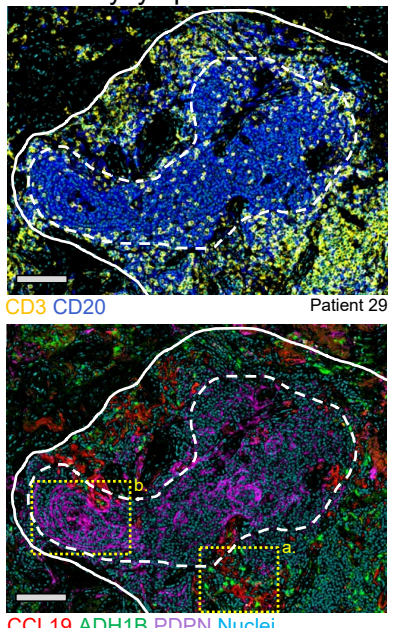

CCL19 ADH1B PDPN Nuclei

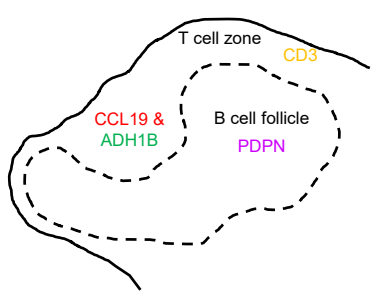

C

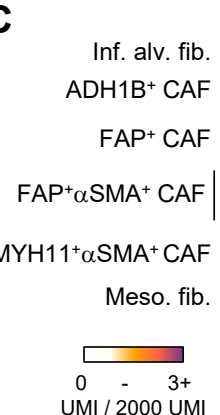

iCAF genes myCAF genes

apCAF genes

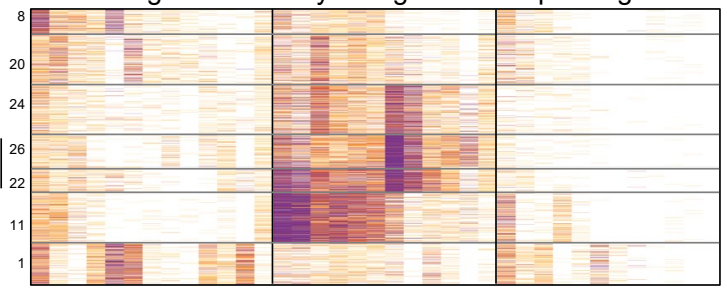

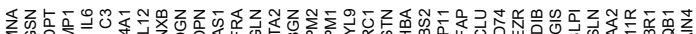


Fig. 2 | Further characterization of CAF subsets in human NSCLC.

A, Stromal cell populations visualized with viSNE in CyTOF. EC, PvC and multiple fibroblast subsets can be distinguished with relatively few markers (CD10, CD31, CD34, CD73, FAP, CD146 and $\alpha$ SMA) B, (upper left panel) Highlighting CCL19 expressing cells within ADH1B ${ }^{+}$ CAF. These cells expressed high amounts of CCL19, CCL21, and VCAM1 and low levels of certain $\mathrm{ADH}_{1 \mathrm{~B}}{ }^{+} \mathrm{CAF}$ genes such as MYH10 and GPC3. (bottom and right panels) Multiplex IHC of a representative tertiary lymphoid structure. Podoplanin (PDPN) and CD20 marks follicular dendritic cells and B cells, respectively, in the B cell follicle, while the T cell zone is identified with CD3 staining. CCL19 and ADH1B staining show $\mathrm{ADH}_{1}{ }^{+}$fibroblasts surrounded by the secreted chemokine CCL19, specifically in the T cell zone. All scale bars are $100 \mu \mathrm{m} . \mathbf{C}$, myCAF, iCAF and apCAF gene signatures (Elyada et al., 2019; Öhlund et al., 2017) projected onto NSCLC CAF clusters. 
expand upon the iCAF, myCAF, and apCAF profiles described in pancreatic tumors(20,22). Our analysis suggests that in human lung tumors, myCAF include both $\mathrm{FAP}^{+} \mathrm{CAF}, \mathrm{FAP}^{+} \alpha \mathrm{SMA}^{+} \mathrm{CAF}$ and $\mathrm{MYH}_{1} 1^{+} \alpha \mathrm{SMA}^{+} \mathrm{CAF}$ highlighting the transcriptomic and spatial complexity of this population (Figure 2C).

ADH1B $^{+}$CAF and FAP ${ }^{+}$CAF stratify NSCLC into two main stromal patterns associated with tumor stage and histology

Analysis of the fibroblast composition as determined by the scRNAseq indicated that low stage tumors were dominated by $\mathrm{ADH}_{1}{ }^{+} \mathrm{CAF}$ with or without $\mathrm{MYH} 11^{+} \alpha \mathrm{SMA}^{+} \mathrm{CAF}$, while higher stage tumors were enriched for $\mathrm{FAP}^{+} \mathrm{CAF}$ and $\mathrm{FAP}^{+} \alpha \mathrm{SMA}^{+} \mathrm{CAF}$ (Figures 3A, 3B, S4A). To test the dichotomy between $\mathrm{ADH}_{1}{ }^{+}$and $\mathrm{FAP}^{+} \mathrm{CAF}$ enrichment, we leveraged a larger cohort of 35170 patient FFPE samples and quantified the tumor area covered by ADH1B and FAP using IHC. This 171 unbiased analysis showed that the stroma of NSCLC is significantly dominated for either ADH1B ${ }^{+}$ 172 or FAP ${ }^{+} \mathrm{CAF}$ (hypergeometric test, $p=0.008$ ) (Table 5, Figure $3 \mathrm{C}$ ). $\mathrm{MYH}_{1} 1^{+} \alpha \mathrm{SMA}^{+} \mathrm{CAF}$ were 173 observed in half of $\mathrm{ADH}_{1}{ }^{+} \mathrm{CAF}$ rich samples (9/18) (Figure 3C) but they were not observed in

$174 \mathrm{FAP}^{+} \mathrm{CAF}$ rich samples, corroborating the scRNAseq analysis that showed a correlation between

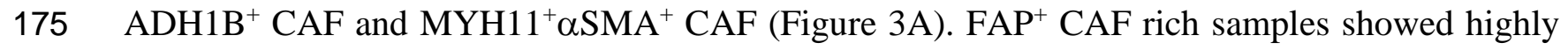
176 variable expression of $\alpha \mathrm{SMA}$ (Figure 3B), in line with the variable ACTA2 expression seen across 177 the scRNAseq $\mathrm{FAP}^{+} \mathrm{CAF}$ clusters (Figure 1C).

179 To study the transcriptional programs behind $\mathrm{ADH}_{1}{ }^{+} \mathrm{CAF}$ and $\mathrm{FAP}^{+} \mathrm{CAF}$ and to better 180 understand their relationship to adjacent tissue fibroblasts, we analyzed gene expression 
Figure 3

A Percent composition T-stage
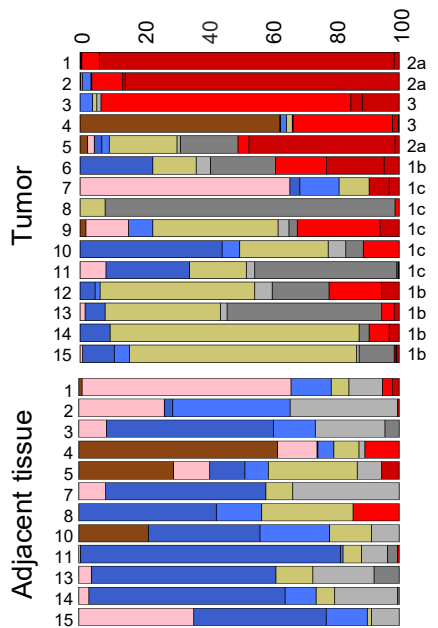

$\mathrm{FAP}^{+} \alpha \mathrm{SMA}^{+} \mathrm{CAF}$ Inf. alveolar fib.

- FAP $^{+}$CAF Alveolar fib.

$\mathrm{MYH}_{11}{ }^{+} \alpha \mathrm{SMA}^{+} \mathrm{CAF} \quad \mathrm{P} / 16^{+} \mathrm{fib}$.

$\mathrm{ADH}_{1 \mathrm{~B}^{+} \mathrm{CAF}} \mathrm{Meso}$. fib.

C

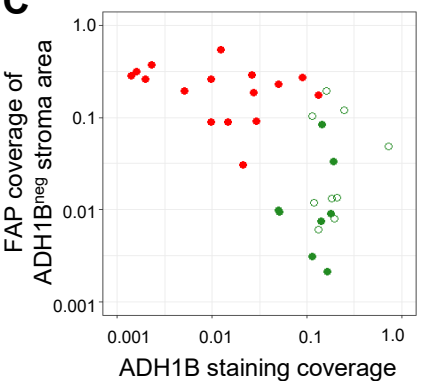

ADH1B ${ }^{+}$CAF-dominant samples

$\mathrm{FAP}^{+} \mathrm{CAF}$-dominant samples

$\begin{aligned} \mathrm{MYH} 11^{+} \alpha \mathrm{SMA}^{+} \mathrm{CAF} & +0 \\ & -\end{aligned}$
B

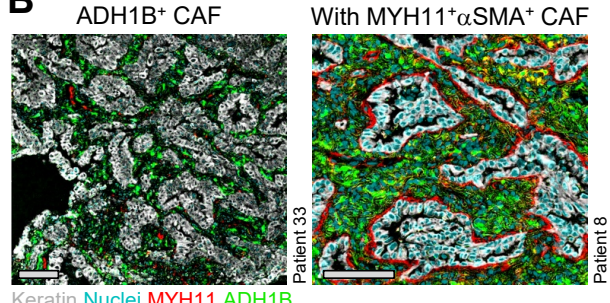

Keratin Nuclei MYH11 ADH1B

$\mathrm{FAP}^{+} \mathrm{CAF}$

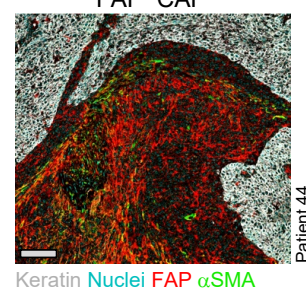

$\mathrm{FAP}^{+} \alpha \mathrm{SMA}^{+} \mathrm{CAF}$

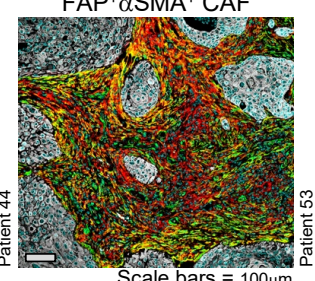

Scale bars $=100 \mathrm{\mu m}$

(2)

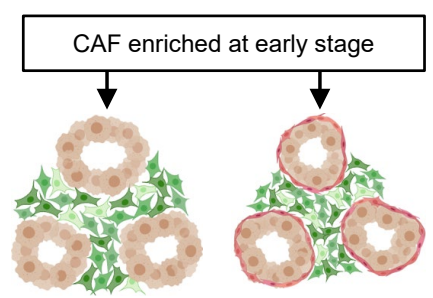

$\mathrm{ADH}_{1 \mathrm{~B}^{+} \mathrm{CAF}}$

$\mathrm{MYH} 11^{+} \alpha \mathrm{SMA}^{+} \mathrm{CAF}$

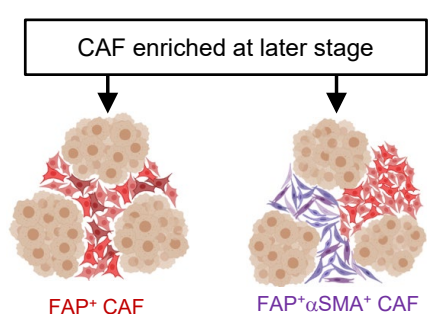

D Key cluster identifying genes

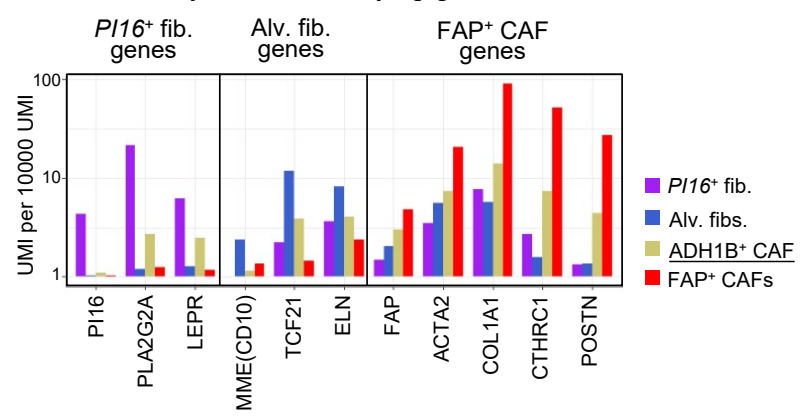

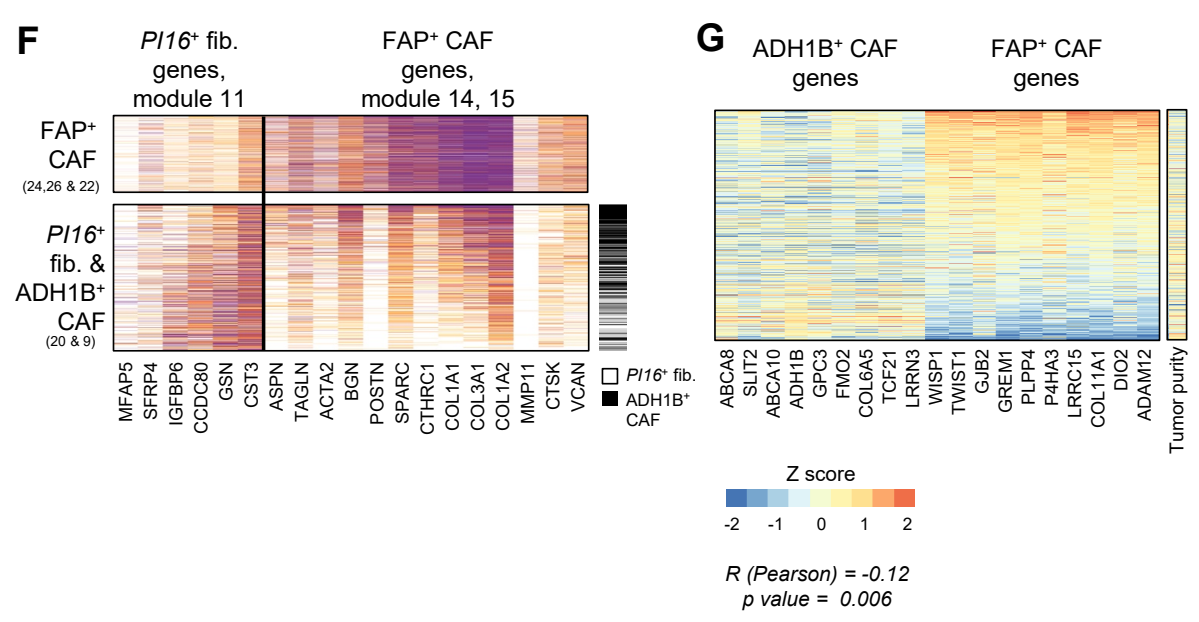

H

Tumor stage

(LUAD) (LUSC)

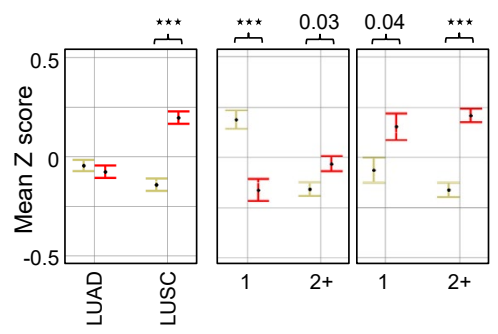

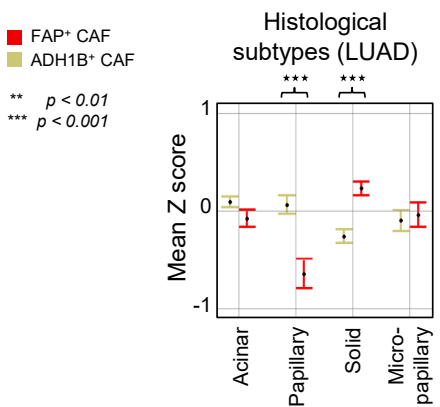


Fig. 3 | ADH1B ${ }^{+}$CAF and FAP ${ }^{+}$CAF stratify NSCLC into two main stromal patterns associated with tumor stage and histology. A, Fibroblast subset composition, displayed by percentages, in individual tumor and adjacent tissue samples from the 15 scRNAseq patients. B, (Top left panels) $\mathrm{ADH}_{1 \mathrm{~B}}{ }^{+} \mathrm{CAF}$ rich patients showing ADH1B presence throughout the stroma. $\mathrm{ADH}_{1 \mathrm{~B}}{ }^{+} \mathrm{CAF}$ rich patients may present with (bottom left panel) or without (top left panel) a distinct single cell layer of $\mathrm{MYH}_{1}{ }^{+} \alpha \mathrm{SMA}^{+} \mathrm{CAF}$ at the tumor border. (Bottom left panels) FAP ${ }^{+}$ CAF rich patients with FAP staining throughout the stroma. The patients shown demonstrate the variable $\alpha \mathrm{SMA}$ presentation in $\mathrm{FAP}^{+}$cells. All scale bars are $100 \mu \mathrm{m}$. (Right panels) Cartoon illustrating the observed presentation of multiple CAF subsets in NSCLC. C, ADH1B and FAP staining in the IHC cohort. ADH1B staining coverage in the stroma is shown on the $\mathrm{X}$ axis. FAP staining coverage in the stroma on regions that did not stain for ADH1B are shown on the Y axis. Tumors show significant preference for either ADH1B or FAP, with less than 5\% coverage of the opposing stain. The 5\% cutoff was selected after performing hypergeometric tests for 10 thresholds, at 5\% increments, between 5\% and 50\%. The Bonferroni correction adjusted p value is 0.008. D, Mean expression of selected genes highlighting $\mathrm{ADH}_{1 \mathrm{~B}}{ }^{+} \mathrm{CAF}$ intermediate expression of $\mathrm{PII6}^{+}$fib., alv. fib., and $\mathrm{FAP}^{+} \mathrm{CAF}$-associated genes. E, Tumor sample with an extensive invasive margin that displays a spectrum of ADH1B to FAP staining. (Zoom, bottom panel) Cells appearing to transition from ADH1B to FAP expression. Top panel scale bar is $200 \mu \mathrm{m}$, bottom panel scale bar is $100 \mu \mathrm{m}$. F, Expression of $\mathrm{PII}^{+}$fib. and $\mathrm{FAP}^{+} \mathrm{CAF}$ module genes in PII6 $^{+}$fib., $\mathrm{ADH}_{1 \mathrm{~B}}{ }^{+} \mathrm{CAF}$, and $\mathrm{FAP}^{+} \mathrm{CAF}$. Based on gene expression patterns, $\mathrm{ADH}^{+} \mathrm{B}^{+} \mathrm{CAF}$ appear to occupy an intermediate state of activation between $\mathrm{PII6}^{+}$fib. and FAP ${ }^{+} \mathrm{CAF}$. G, Relative expression, displayed by $\mathrm{Z}$ score, of $\mathrm{ADH}^{+} \mathrm{B}^{+} \mathrm{CAF}$ and $\mathrm{FAP}^{+} \mathrm{CAF}$-associated genes in TCGA LUAD bulk-RNAseq samples. $\mathrm{ADH}^{+} \mathrm{CAF}$ and $\mathrm{FAP}^{+} \mathrm{CAF}$ genes are significantly 
anticorrelated (Pearson) $R=-0.12$ and $p=0.006$. The sample tumor nuclei count is used as a proxy of tumor purity and shows a relatively even distribution. H, TCGA LUAD mean Z score and standard error of mean (SEM) of $\mathrm{ADH} \mathrm{B}^{+} \mathrm{CAF}$ and $\mathrm{FAP}^{+} \mathrm{CAF}$ gene signatures stratified by tumor subtype (left and middle panels) or stage (right panel). Z score calculation is listed in methods and significance is calculated by independent $\mathrm{t}$ test (right panel). 


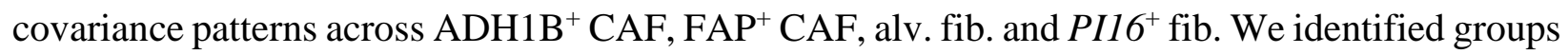
of co-expressed genes (gene modules) with distinct expression patterns across these fibroblast populations (Figure S4B). FAP ${ }^{+} \mathrm{CAF}$ upregulated activation genes (modules 14, 15) including multiple collagen genes (COL1A1,COL3A1) that contribute to tissue stiffness(38) and other ECM genes such as biglycan $(B G N)$ that can promote tissue mineralization (39) (Figure S4B). FAP ${ }^{+}$ CAF expressed low levels of the alv. fib. genes, including the fibroblast transcription factor TCF21 (40), the marker $M M E(\mathrm{CD} 10)$ as well as the ECM gene elastin (ELN) which is critical for normal lung physiology(41) (Figure 2D). $\mathrm{ADH}^{+} \mathrm{B}^{+} \mathrm{CAF}$ expressed intermediate levels of $\mathrm{FAP}^{+} \mathrm{CAF}$ activation genes (Figure 3D), and a subset of samples showed a gradient of $\mathrm{ADH}^{+} \mathrm{B}^{+} \mathrm{CAF}$ to $\mathrm{FAP}^{+}$ CAF from the invasive margin to the tumor center (Figure 3E) with some cells co-expressing both markers, suggesting that $\mathrm{ADH}_{1} \mathrm{~B}^{+} \mathrm{CAF}$ represent a range of lowly activated fibroblasts. $\mathrm{ADH} 1 \mathrm{~B}^{+}$ CAF also shared genes with both $P I 16^{+}$fib. and alv. fib., which may point towards both lung fibroblast types as their potential cellular sources (Figures 3D, S4C). Interestingly, the scRNAseq data showed that ADH1B CAF cells express a gradient of $\mathrm{FAP}^{+} \mathrm{CAF}$ and $\mathrm{PII6}^{+}$fib. genes from cells with high expression of PII6 genes and low FAP genes to cells with low PI16 genes and high

196 FAP genes. This further supports the hypothesis that $\mathrm{ADH}^{+} \mathrm{B}^{+} \mathrm{CAF}$ are a lowly activated form of 197 fibroblast and may derive from PII $^{+}$fib. (Figure 3F). by selecting for genes with highly specific expression in their corresponding CAF populations in 201 contrast with all other cell types. With these signatures we scored the Cancer Genome Atlas 202 (TCGA) lung adenocarcinoma (LUAD) samples by their expression of $\mathrm{ADH} \mathrm{B}^{+} \mathrm{CAF}$ and $\mathrm{FAP}^{+}$ 203 CAF genes and revealed an anticorrelation between the two scores $(p=0.006)$ (Figures 3G, S4D, 
204 Table 6), supporting the two distinct CAF profiles observed across NSCLC patients in scRNAseq

205 and histology (Figures 3A-C) (42). Analysis of tumor purity, estimated by tumor nuclei abundance,

206 did not reveal clear association with $\mathrm{ADH}^{+} \mathrm{B}^{+} \mathrm{CAF}$ or $\mathrm{FAP}^{+} \mathrm{CAF}$ genes (Figure 3G), confirming

207 that contaminating adjacent tissue was not a major contributor to the $\mathrm{ADH}_{1 \mathrm{~B}}{ }^{+} \mathrm{CAF}$ signal. Further

208 analysis of TCGA data showed that $\mathrm{ADH} \mathrm{B}^{+} \mathrm{CAF}$ genes were significantly increased in stage 1

209 tumors, LUAD and the papillary LUAD subtype, whereas $\mathrm{FAP}^{+} \mathrm{CAF}$ were enriched in later stage

210 tumors, LUSC, and the LUAD solid subtype (Figure 3H). LUAD across tumor stages confirmed

211 our observation that $\mathrm{ADH}_{1} \mathrm{~B}^{+} \mathrm{CAF}$ and $\mathrm{FAP}^{+} \mathrm{CAF}$ were correlated with lower and higher stage

212 tumors, respectively. Similar associations were observed in our in-house FFPE cohort (Table 1).

213 Altogether, we showed that $\mathrm{ADH}_{1}{ }^{+}$and $\mathrm{FAP}^{+} \mathrm{CAF}$ phenotypes were correlated with tumor stage

214 and clinically relevant histological subtypes $(42,43)$, suggesting that molecular characterization of

215 fibroblasts could refine clinical categorization of NSCLC tumors.

216

$217 \mathrm{ADH1B}^{+}$CAF and FAP ${ }^{+}$CAF correlate with immune cell composition and not with $\mathbf{T}$ cell

218 localization

219

220 Given the data showing that CAF contribute to regulating tumor immunity $(11,12,44)$ we

221 investigated the different ligands expressed by CAF populations found in human NSCLC (Figure

222 4A). Increased expression of the cytokines IL34 and CSF 1 suggested macrophage regulation (45)

223 by $\mathrm{ADH}_{1}{ }^{+} \mathrm{CAF}$, whereas $\mathrm{FAP}^{+} \mathrm{CAF}$ might attract eosinophils/basophils via CCL11 (46), as well

224 as $\mathrm{CCR}^{+} \mathrm{T}$ cells and monocytes through CCL3 and CCL5 chemokines (47-49). Notably, the high

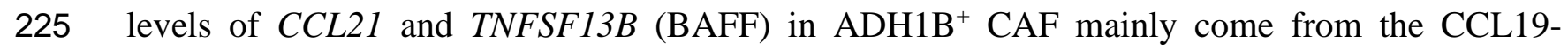

226 expressing $\mathrm{ADH}_{1 \mathrm{~B}}{ }^{+}$cells specifically found in TLS (Figures 4A, 2B), likely contributing to naïve 
A Ligands

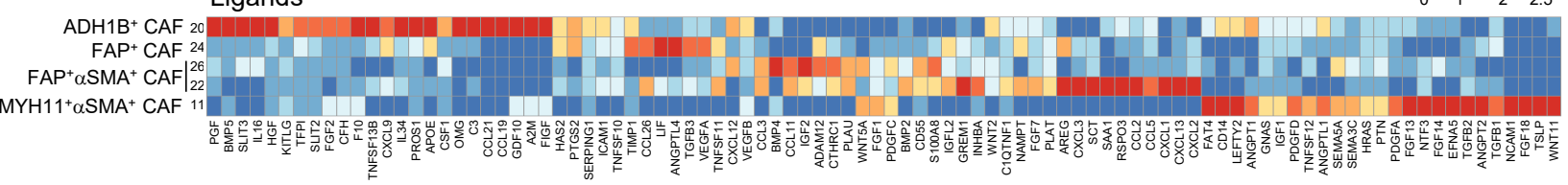

B

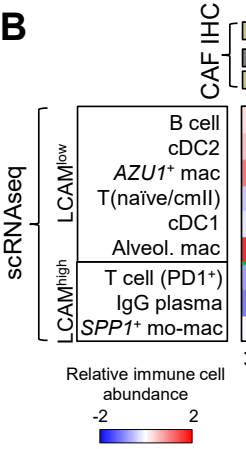

D

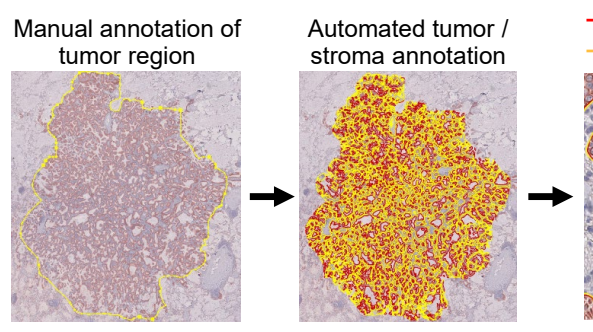

E

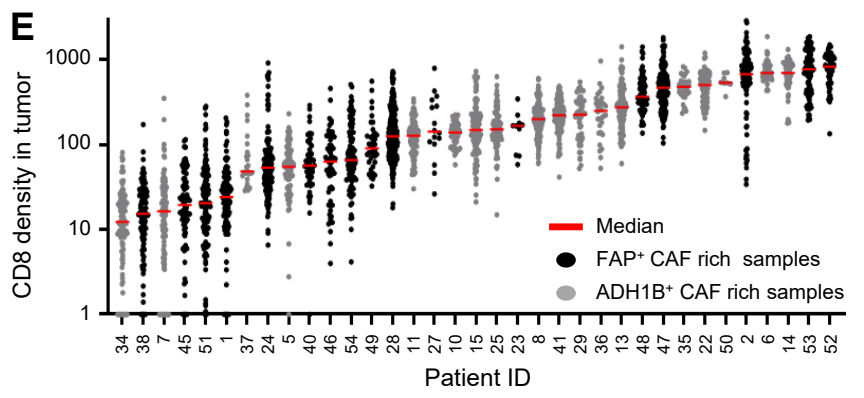

C

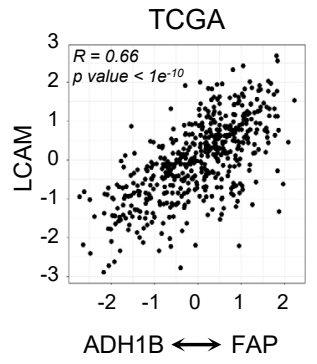

Tumor / stroma annotations transferred to CD3 / CD8 image

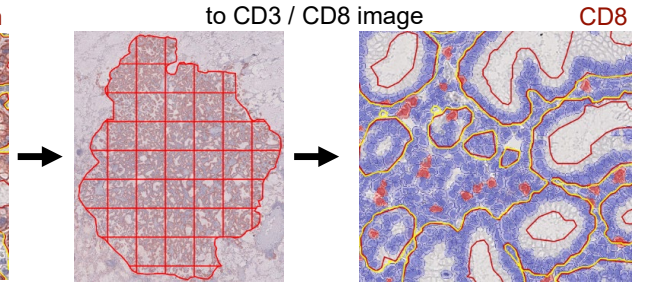

Dividing tissue into tiles
$\mathrm{CD}^{+}$or $\mathrm{CD}^{+}$

positive cell detection

F $\mathrm{CD}^{+}$tumor / $\mathrm{CD}^{+}$tumor /

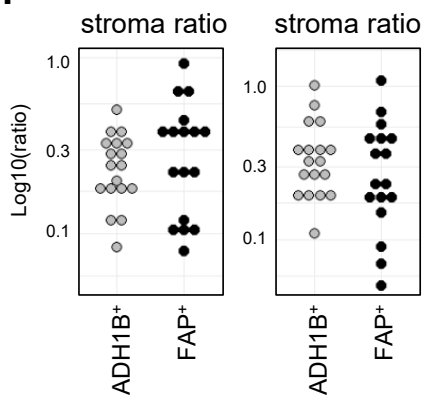




\section{Fig. 4 | ADH1B ${ }^{+}$CAF and FAP ${ }^{+}$CAF correlate with immune cell composition and not with}

T cell localization. A, Gene expression over mean of highly variable immunomodulatory ligands in CAF clusters. B, Immune composition of scRNAseq tumor samples from (Leader et al., 2021). CAF phenotype is identified by IHC on the matched FFPE samples and then used to stratify samples. The relative abundance of each cell population within its respective compartment, i.e.; $\mathrm{PD}^{+} \mathrm{T}$ cells amongst all $\mathrm{T}$ cells, is calculated and then scaled across all tumors for the respective $\mathrm{Z}$ score value. LCAM score is significantly correlated with CAF phenotype (Pearson) $R=0.62, p$ $=0.01 . \mathbf{C}$, Estimating the correlation between CAF phenotype and LCAM in TCGA LUAD samples. Each patients' mean $\mathrm{ADH} \mathrm{B}^{+} \mathrm{CAF}$ gene signature is subtracted from their mean $\mathrm{FAP}^{+}$ CAF gene signature and the resulting values are correlated with estimate LCAM score. The corresponding Pearson correlation values are shown. D, Schematic of QuPath methodology for tiling and $\mathrm{T}$ cell quantification. $\mathbf{E}, \mathrm{CD}^{+}$cell infiltration into tumor nests in each patient (columns). Each point represents an individual $1000 \mu \mathrm{m} \times 1000 \mu \mathrm{m}$ tile (all other tiling is $500 \mu \mathrm{m} \times 500 \mu \mathrm{m}$ ). F, IHC quantification of $\mathrm{CD}^{+}$or $\mathrm{CD}^{+}$cells per $\mathrm{mm}^{2}$ in the tumor and the tumor / stroma $\mathrm{CD}^{+}$ or $\mathrm{CD}^{+}$cells per $\mathrm{mm}^{2}$ ratio. Tumor samples are stratified by their stroma profile (ADH1B ${ }^{+} \mathrm{CAF}$ rich or $\mathrm{FAP}^{+} \mathrm{CAF}$ rich) and no significant difference was observed. 
$227 \mathrm{~T}$ cell attraction and B cell survival in these structures (37). MYH1 $11^{+} \alpha \mathrm{SMA}^{+} \mathrm{CAF}$ expressed

228 increased levels of TSLP (thymic stromal lymphopoietin) which can stimulate the maturation of

229 immune cells that express both $I L 7 R$ and $C R L F 2$ genes forming the heterodimeric TSLP receptor,

230 such as certain dendritic cells (50). To further investigate the contribution of different CAF

231 populations to shaping the immune microenvironment, we used multiplex imaging on FFPE tissue

232 to histologically profile the CAF subset composition of a large cohort of NSCLC samples that we

233 had previously studied using scRNAseq of purified immune cells(29). We demonstrated a

234 significant association (Pearson, $R=0.62, p=0.01$ ) between the presence of $\mathrm{FAP}^{+} \mathrm{CAF}$ and the

235 enrichment of inflammatory $S P P 1^{+}$monocyte-derived macrophages, $\mathrm{IgG}^{+}$plasma cells, and $\mathrm{PD} 1^{+}$

236 T cells (Figure 4B). These immune cell types were recently described as part of a cellular module

237 termed Lung Cancer Activation Module (LCAM)(29). We then validated this CAF-immune

238 association in the TCGA LUAD cohort. There was a significant correlation between CAF

239 phenotype and the LCAM score $\left(R=0.66, p<1 e^{-10}\right)$, supporting that $\mathrm{FAP}^{+}$CAF rich samples are

240 linked to more inflammatory and activated immune cells, LCAM ${ }^{\text {high }}$, in LUAD (Figure 4C).

242 Given that the spatial distribution of $\mathrm{T}$ cells is a predictor of clinical response to immune

243 checkpoint blockade (51), we used an unbiased cell quantification method to measure $\mathrm{T}$ cell

244 infiltration in the tumor nests and identified a wide range of infiltration levels across the cohort

245 (Figures 4D-F). Importantly, there was no observed association between any of $\mathrm{ADH}^{+} \mathrm{B}^{+}$and

$246 \mathrm{FAP}^{+} \mathrm{CAF}-$ rich profiles and $\mathrm{CD}^{+}$and $\mathrm{CD}^{+} \mathrm{T}$ cell localization (Figures 4E-F, Table 7). Taken

247 together, $\mathrm{ADH}_{1 \mathrm{~B}}{ }^{+} \mathrm{CAF}$ and $\mathrm{FAP}^{+} \mathrm{CAF}$ stratify tumor lesions by two levels of fibroblast activation

248 and correlate with the immune phenotype, but not with T cell spatial distribution. 
$\mathrm{MYH11}^{+} \alpha \mathrm{SMA}^{+} \mathrm{CAF}$ are correlated with decreased $\mathrm{T}$ cell infiltration in tumor nests

252 The lack of correlation between $\mathrm{ADH}_{1 \mathrm{~B}^{+}} \mathrm{CAF}$ or $\mathrm{FAP}^{+} \mathrm{CAF}$ with $\mathrm{CD}^{+}$or $\mathrm{CD}^{+} \mathrm{T}$ cell infiltration

253 contrasts with the general idea that activated fibroblasts orchestrate $\mathrm{T}$ cell exclusion, raising the

254 hypothesis that fibroblast subsets other than $\mathrm{ADH}^{+} \mathrm{B}^{+}$or $\mathrm{FAP}^{+} \mathrm{CAF}$ could be involved. To

255 investigate if $\mathrm{MYH} 11^{+} \alpha \mathrm{SMA}^{+} \mathrm{CAF}$, which form a single cell layer around tumor nests in a fraction

256 of early-stage tumors, could also impact T cell tumor infiltration, we subdivided stage 1 patients

257 based on the presence of $\mathrm{MYH} 1^{+} \alpha \mathrm{SMA}^{+} \mathrm{CAF}$ at the tumor border (Figure 5A). In tumor lesions

258 containing $\mathrm{MYH}_{1} 1^{+} \alpha \mathrm{SMA}^{+} \mathrm{CAF}$, the tumor-to-stroma ratio of infiltrating $\mathrm{CD} 3^{+}$or $\mathrm{CD}^{+}$cells

259 was significantly lower (Figure 5B, right graphs), consistent with a decreased infiltrating $\mathrm{CD}^{+}$or

$260 \mathrm{CD}^{+} \mathrm{T}$ cell density in the tumor (Figure 5B, left graphs) (Table 7). In addition, their high

261 expression of $T G F B 1$ and TGFB2 (Figure 4A) is in line with previous findings linking TGF $\beta$ and

262 T cell exclusion $(6,52)$. These results suggested that $\mathrm{MYH} 11^{+} \alpha \mathrm{SMA}^{+} \mathrm{CAF}$, with their peri-tumoral

263 location, may decrease T cells infiltration into tumor nests.

265 Within our cohort, MYH $11^{+} \alpha \mathrm{SMA}^{+} \mathrm{CAF}$ were found enriched in LUAD samples, especially in

266 the acinar/papillary subtypes, while neither the solid subtype of LUAD, nor LUSC samples

267 contained MYH1 $11^{+} \alpha \mathrm{SMA}^{+} \mathrm{CAF}$ lining tumor nests (Figure 5E). Interestingly, the IHC image bank

268 of the Human Protein Atlas showed that a similar peritumoral MYH11 staining pattern as one layer

269 was observed in a fraction of samples of pancreatic and breast cancer (Figure 5F), suggesting that

270 these CAF may be present in additional cancer types. 


\section{Figure 5}

A Early-stage tumors (stage 1) No MYH11+ CAF
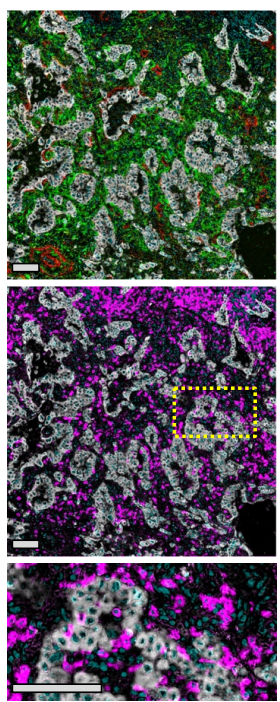

Keratin Nuclei Patient 26 MYH11 ADH1B CD3
With MYH11+ CAF
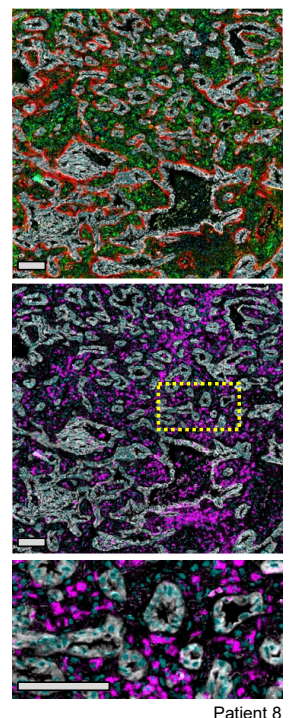

Scale bars $=100 \mu \mathrm{m}$
B
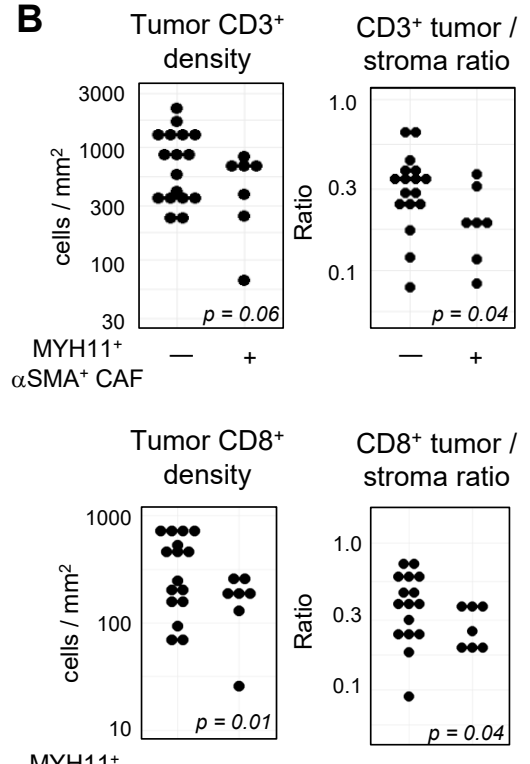
$\mathrm{MYH}^{+} 1^{+}$
$\alpha \mathrm{SMA}^{+} \mathrm{CAF}$
C Tumor $\mathrm{CD}^{+}$densitv

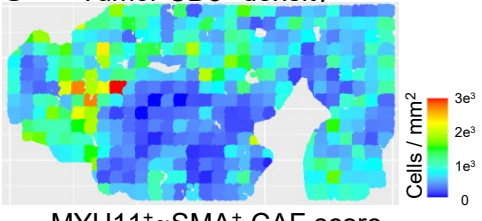

$\mathrm{MYH}_{11}{ }^{+} \alpha \mathrm{SMA}^{+} \mathrm{CAF}$ score
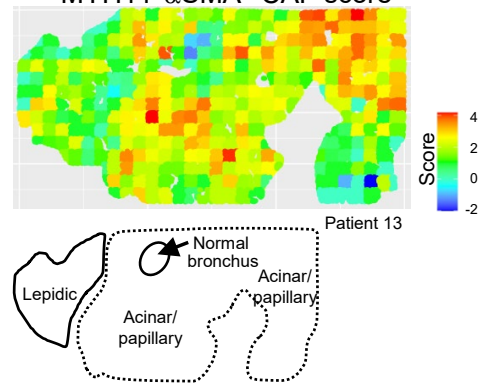

D

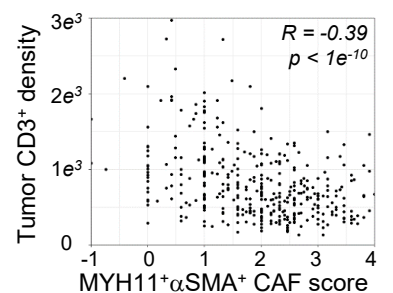

E
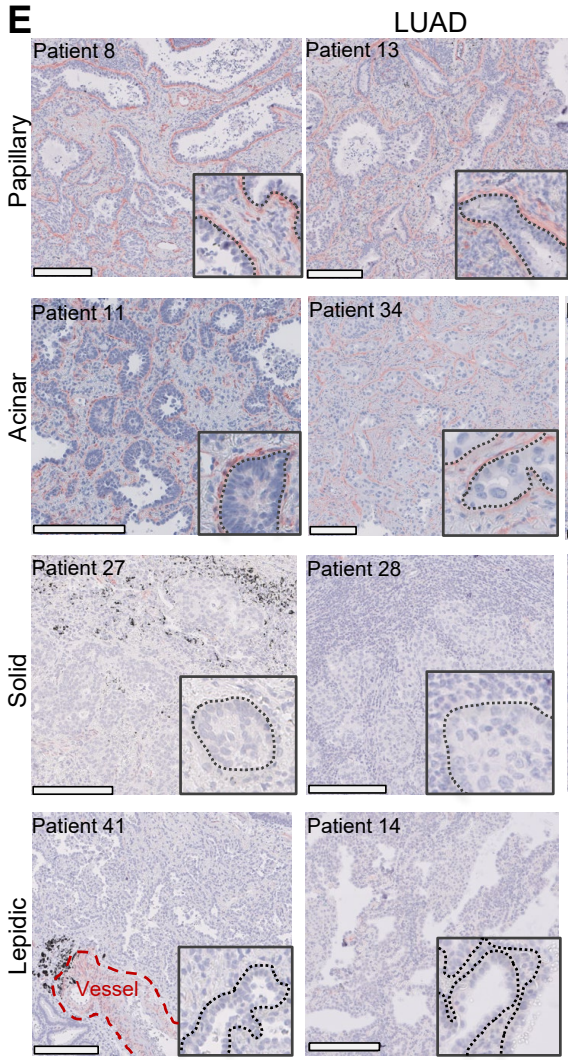

Patient 34

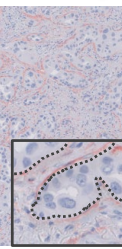

Patient 28

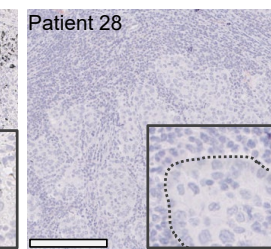

Patient 14

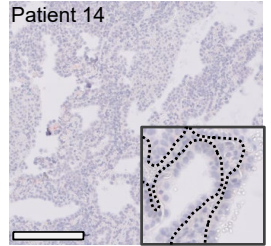

MYH11

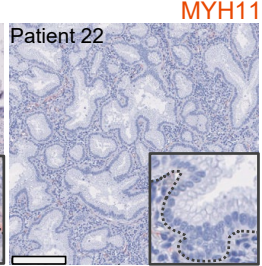

Patient 15

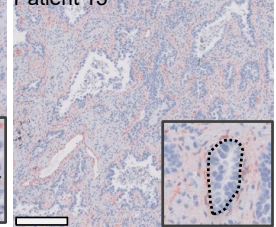

Patient 6

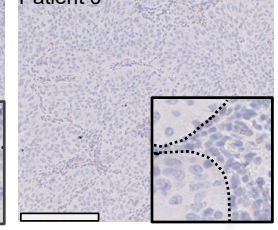

Patient 40

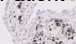

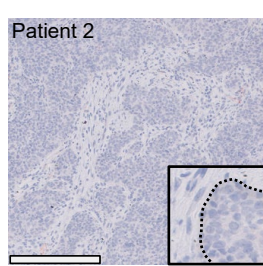

LUSC
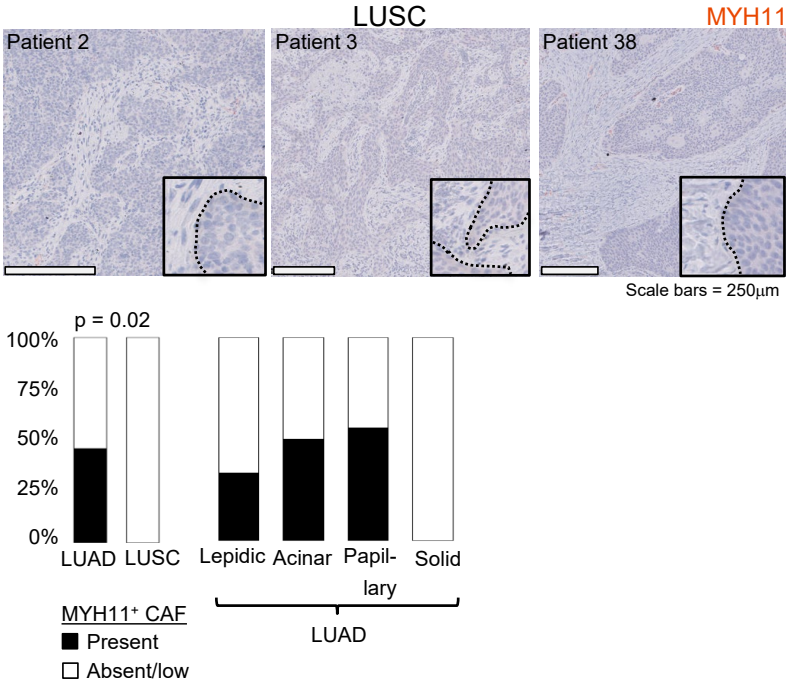

F Pancreatic cancer

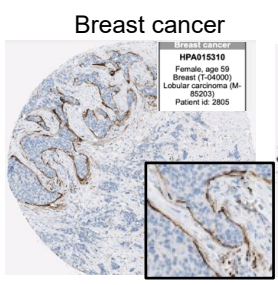

Lung cancer

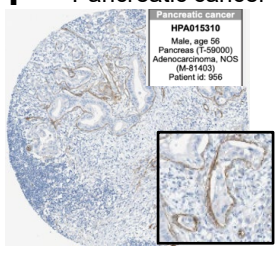

MYH11 Staining from The Human Protein Atlas

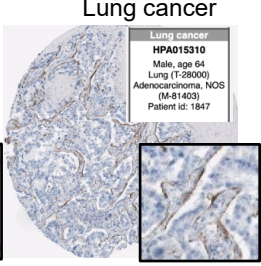




\section{Fig. 5 | MYH11 ${ }^{+} \alpha \mathrm{SMA}^{+} \mathrm{CAF}$ are correlated with decreased $\mathrm{T}$ cell infiltration in tumor nests.}

A, Representative examples of IHC stains from NSCLC tumors with and without MYH1 $1^{+} \alpha \mathrm{SMA}^{+}$ CAF present, showing $\mathrm{CD}^{+}$cell exclusion from tumor nests when $\mathrm{MYH} 11^{+} \alpha \mathrm{SMA}^{+} \mathrm{CAF}$ are present. B, The presence or absence of $\mathrm{MYH} 11^{+} \alpha \mathrm{SMA}^{+} \mathrm{CAF}$ demonstrates significant differences in tumor infiltrating $\mathrm{CD}^{+}$or $\mathrm{CD}^{+}$cells per $\mathrm{mm}^{2}$ (left) and the ratio of $\mathrm{CD}^{+}$or $\mathrm{CD}^{+}$cells per $\mathrm{mm}^{2}$ in the tumor versus stroma (right). Only early stage (tumor stage 1 ) patients were included to eliminate bias due to $\mathrm{MYH} 11^{+} \alpha \mathrm{SMA}^{+} \mathrm{CAF}$ rarely being found at later stage. $\mathrm{C}, 500 x 500 \mu \mathrm{m}$ tiles of both $\mathrm{MYH} 11^{+} \alpha \mathrm{SMA}^{+} \mathrm{CAF}$ score, estimating tumor proximity of $\mathrm{MYH}^{+} 1^{+} \alpha \mathrm{SMA}^{+}$cells by quantifying their enrichment within $10 \mu \mathrm{m}$ from tumor cells versus regions $20 \mu \mathrm{m}-30 \mu \mathrm{m}$ from tumor cells, and tumor infiltrating $\mathrm{CD}^{+}$cells per $\mathrm{mm}^{2}$. (Bottom panel) Histological scoring of a tumor lesion highlighting that a high $\mathrm{MYH} 11^{+} \alpha \mathrm{SMA}^{+} \mathrm{CAF}$ score is associated more with acinar/papillary phenotype, rather than lepidic. D, Quantification of results in F, a high $\mathrm{MYH}_{11^{+}} \alpha \mathrm{SMA}^{+} \mathrm{CAF}$ score is significantly anti-correlated (Pearson) with the number of tumor infiltrating $\mathrm{CD}^{+}$cells relative to the stroma. E, Representative images of MYH11 staining in multiple pathologies and histological subtypes. All scale bars are $250 \mu \mathrm{m}$. (Barplot) $\mathrm{MYH}_{11}{ }^{+} \alpha \mathrm{SMA}^{+} \mathrm{CAF}$ distribution in different pathologies and histological subtypes in NSCLC. F, MYH11 staining from The Human Protein Atlas. 
271 While most tumor lesions were characterized by either high or low $\mathrm{MYH}_{1} 1^{+} \alpha \mathrm{SMA}^{+} \mathrm{CAF}$

272 presence, a fraction of tumors showed local heterogeneity. We assessed the intensity of CAF

273 barrier at the tumor boundary in $500 \mu \mathrm{m}$-by-500 $\mu \mathrm{m}$ tiles using the abundance of MYH $11^{+} \alpha \mathrm{SMA}^{+}$

274 cells in the stroma close to $(<10 \mu \mathrm{m})$ versus distant from $(20 \mu \mathrm{m}-30 \mu \mathrm{m})$ tumor cells, which is

275 referred to as the MYH11 ${ }^{+} \alpha \mathrm{SMA}^{+} \mathrm{CAF}$ score (Figures 5C-D, S5). This automated analysis found

276 that locations where $\mathrm{MYH} 11^{+} \alpha \mathrm{SMA}^{+} \mathrm{CAF}$ were present had significantly lower tumor $\mathrm{T}$ cell

277 density in two independent samples, highlighting that local spatial organization may be driving

278 inter-tumor differences (Figure 5D). Additionally, histological analysis of the tumor lesion by a

279 pathologist found that regions with high $\mathrm{MYH}_{1} 1^{+} \alpha \mathrm{SMA}^{+} \mathrm{CAF}$ score were predominantly

280 acinar/papillary, and lepidic regions at the tumor edge had a lower score (Figure 5C, bottom panel).

281 Altogether, these results show that $\mathrm{MYH} 11^{+} \alpha \mathrm{SMA}^{+} \mathrm{CAF}$ are a single layer of elongated cells

282 associated with $\mathrm{T}$ cell marginalization both across NSCLC tumor samples and within tumor

283 lesions.

284

285

$\mathrm{FAP}^{+} \alpha \mathrm{SMA}^{+} \mathrm{CAF}$ define regions of poor $\mathrm{T}$ cell infiltration within tumor lesions and are

coupled with dense ECM deposition

288 Spatial analysis of $\mathrm{FAP}^{+} \mathrm{CAF}$ rich samples revealed that $\mathrm{FAP}^{+} \alpha \mathrm{SMA}^{+} \mathrm{CAF}$, a subset of $\mathrm{FAP}^{+}$ 289 CAF in scRNAseq, could also explain $\mathrm{CD}^{+}$and $\mathrm{CD}^{+} \mathrm{T}$ cell infiltration within tumors. We 290 measured $\alpha$ SMA coverage and T cell density in the stroma in $500 \mu \mathrm{m}$-by-500 $\mu \mathrm{m}$ sections across 291 the tumor lesion (Figure 6A) and revealed that regions dense in $\alpha$ SMA are poorly infiltrated by $\mathrm{T}$ 292 cells $\left(R=-0.48, p=1 e^{-10}\right)$ (Figure 6B). This anticorrelation was replicated across different tumors 293 (Figures S6A-C) and suggested that $\mathrm{FAP}^{+} \alpha \mathrm{SMA}^{+} \mathrm{CAF}$ directly restrict $\mathrm{T}$ cell motility. Notably, a 


\section{Figure 6}

A Stroma $\mathrm{CD}^{+}$density

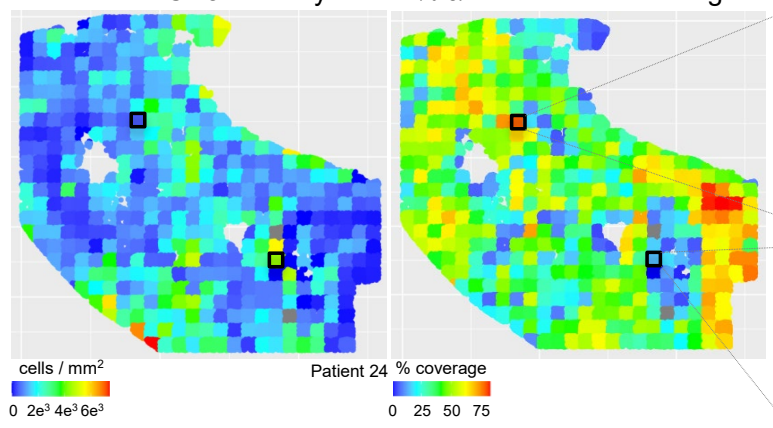

Scale bars $=100 \mu \mathrm{m}$

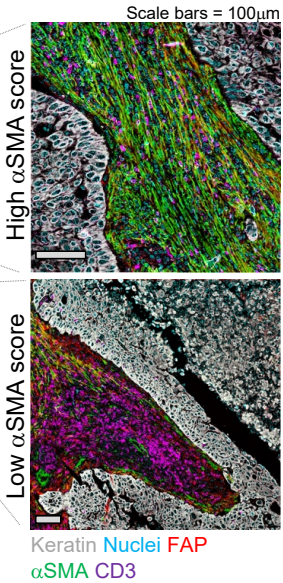

B

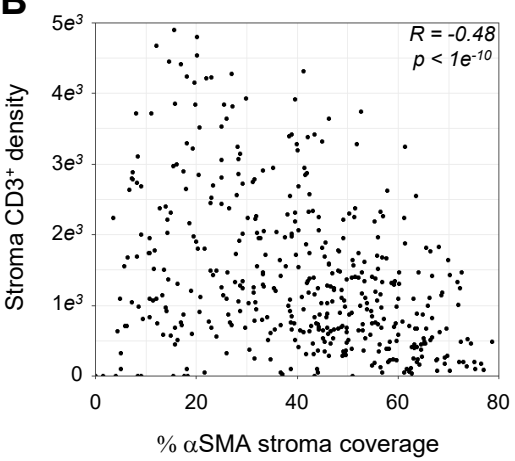

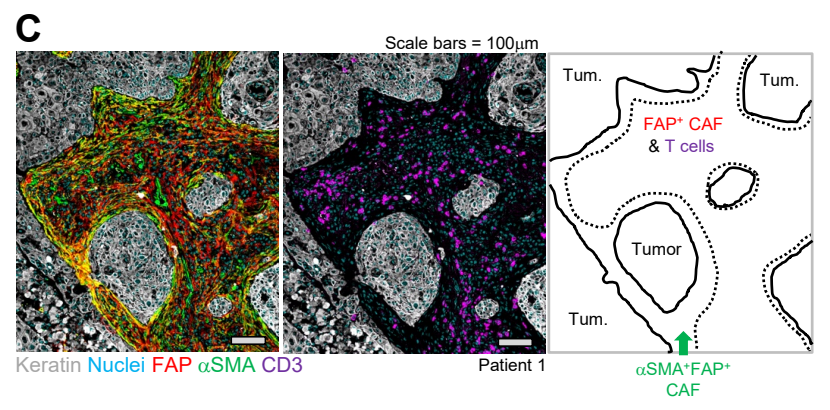
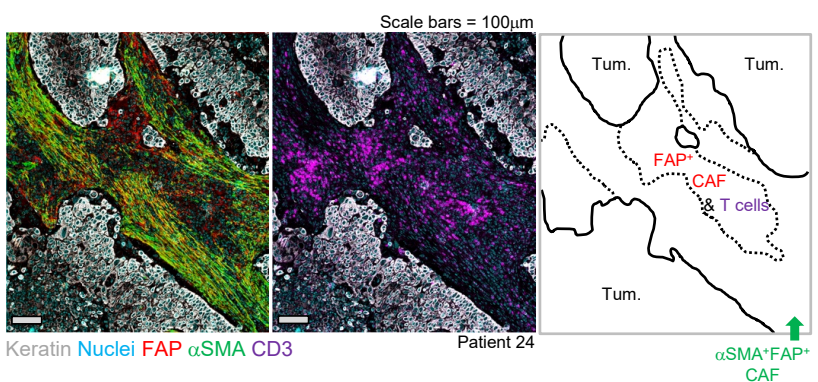

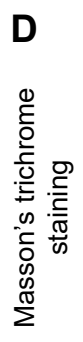

Samples with $\mathrm{FAP}^{+} \alpha \mathrm{SMA}^{+} \mathrm{CAF}$ lining tumor nests

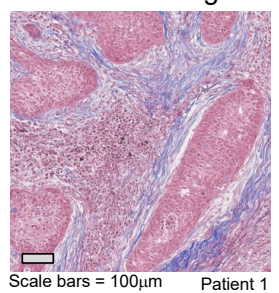

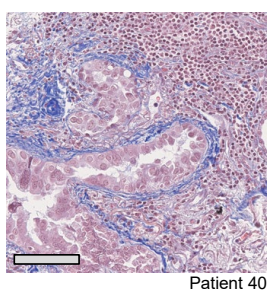

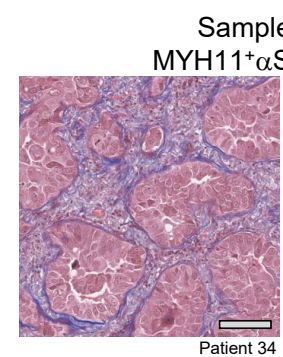

es with

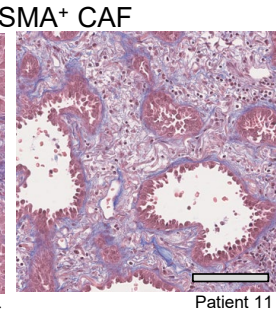

No MYH11 $1{ }^{+} \alpha \mathrm{SMA}^{+}$ or $\mathrm{FAP}^{+} \alpha \mathrm{SMA}^{+} \mathrm{CAF}$

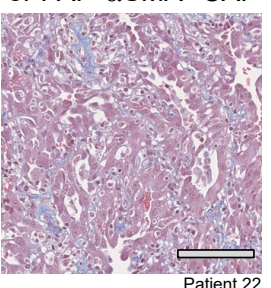

$\mathbf{E}$

Extracellular matrix

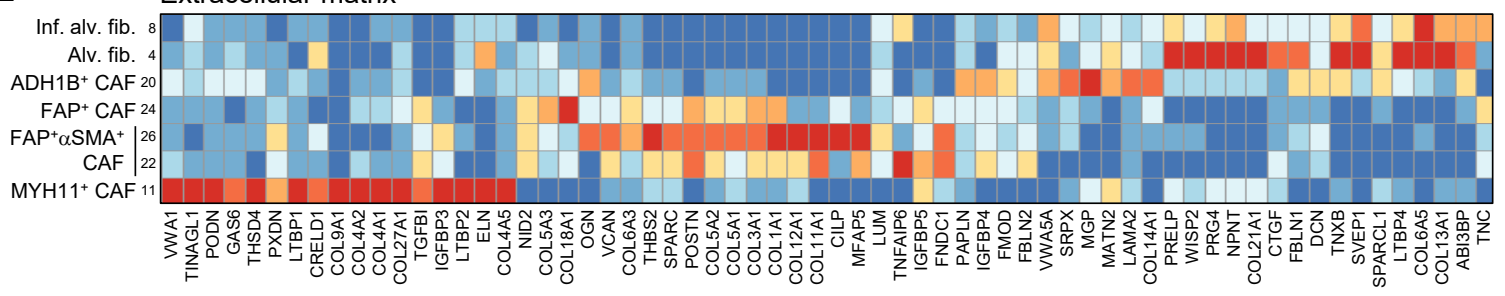


Fig. $6 \mid \mathrm{FAP}^{+} \alpha \mathrm{SMA}^{+} \mathrm{CAF}$ define patterns of poor $\mathrm{T}$ cell infiltration within tumor lesions. A, (Left panel) Intra-tumoral heterogeneity of $\alpha$ SMA coverage (middle panel) and CD $3^{+}$cell density in the stroma in 500x500 $\mu \mathrm{m}$ tiles. (Right panel) Representative examples of tiles showing regions with high or low levels of $\alpha$ SMA. B, Quantification of $\alpha \mathrm{SMA}$ coverage and $\mathrm{CD} 3^{+}$density in each tile (points) as defined in A, showing a significant anticorrelation (Pearson) of $\alpha$ SMA coverage and $\mathrm{CD}^{+}$cell density. $\mathrm{C}$, Dense $\alpha \mathrm{SMA}$ staining at tumor border associates with decreased $\mathrm{CD}^{+}$ cell abundance. The green arrow highlights border regions with high $\alpha \mathrm{SMA}$ and low $\mathrm{CD}^{+}$cells. D, Masson's trichrome stains highlighting increased ECM at the tumor boundary in samples containing $\mathrm{MYH}_{1} 1^{+} \alpha \mathrm{SMA}^{+}$or $\mathrm{FAP}^{+} \alpha \mathrm{SMA}^{+} \mathrm{CAF}$. E, Averaged gene expression of highly variable ECM genes in CAF clusters. 
294 high fraction of $\mathrm{FAP}^{+} \mathrm{CAF}$ rich samples presented with several layers of $\mathrm{FAP}^{+} \alpha \mathrm{SMA}^{+} \mathrm{CAF}$ lining tumor nests that delineated regions devoid of T cells (Figure 6C), and this was corroborated by the finding that regions of high stromal $\alpha$ SMA coverage were associated with low $\mathrm{T}$ cell infiltration in the tumor nests (Figure S6C). In addition, inter-tumor aSMA heterogeneity showed a trend towards an anti-correlation with T cell infiltration in tumor nests (Figures S6D-E).

Based on prior studies showing that the ECM plays a role in $\mathrm{T}$ cell exclusion and immunosuppression(7,53,54), we postulated that $\mathrm{FAP}^{+} \alpha \mathrm{SMA}^{+} \mathrm{CAF}$ may also express a specific ECM profile involved in regulating T cell localization. Masson's trichrome staining revealed a high density of fiber deposition at the tumor border in both $\mathrm{MYH} 11^{+} \alpha \mathrm{SMA}^{+}$and $\mathrm{FAP}^{+} \alpha \mathrm{SMA}^{+}$ CAF-containing tumor lesions, suggesting that $\mathrm{FAP}^{+} \alpha \mathrm{SMA}^{+} \mathrm{CAF}$ are depositing a fibrillar barrier limiting $\mathrm{T}$ cell access to tumor cells (Figure 6D). Analysis of the scRNAseq showed that FAP ${ }^{+}$ $\mathrm{aSMA}^{+} \mathrm{CAF}$ expressed high levels of the fibrillar collagen COL11A1 (55), and COL12A1 (Figure basement membrane collagens, COLAA1 and COLAA2, which are found lining vessels and various $\mathrm{MYH} 11^{+} \alpha \mathrm{SMA}^{+} \mathrm{CAF}$ are two distinct types of fibroblasts forming the physical barrier of fibers which was previously observed around tumor nests and shown to restrict $\mathrm{T}$ cell infiltration into

313 tumor islets in viable slices of human NSCLC lesions (7). Thus, the specific spatial distribution of

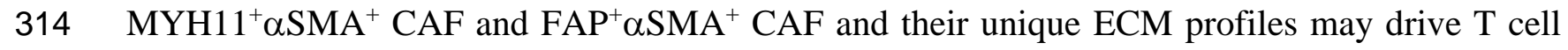
315 exclusion in NSCLC and represent potential therapeutic targets, leading to a proposed model that 316 refines the CAF landscape in human NSCLC (Figure 7). 
A

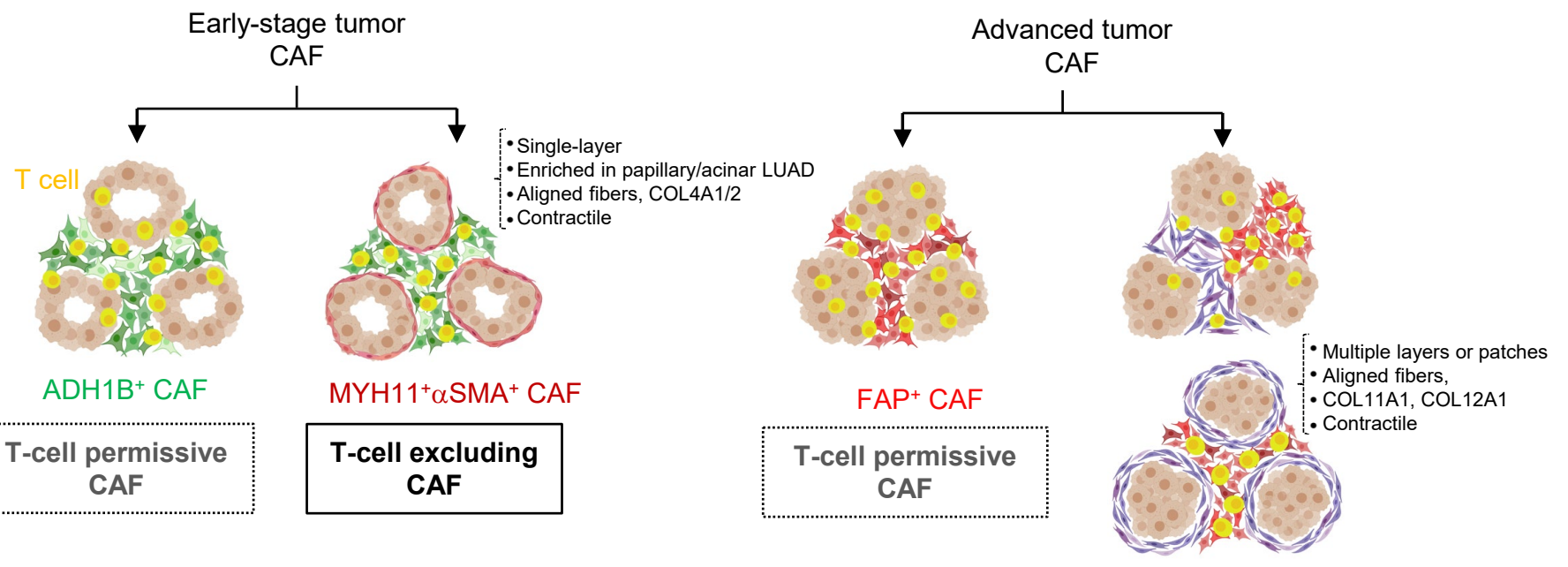

$\mathrm{FAP}^{+} \alpha \mathrm{SMA}^{+} \mathrm{CAF}$

T-cell excluding CAF

B

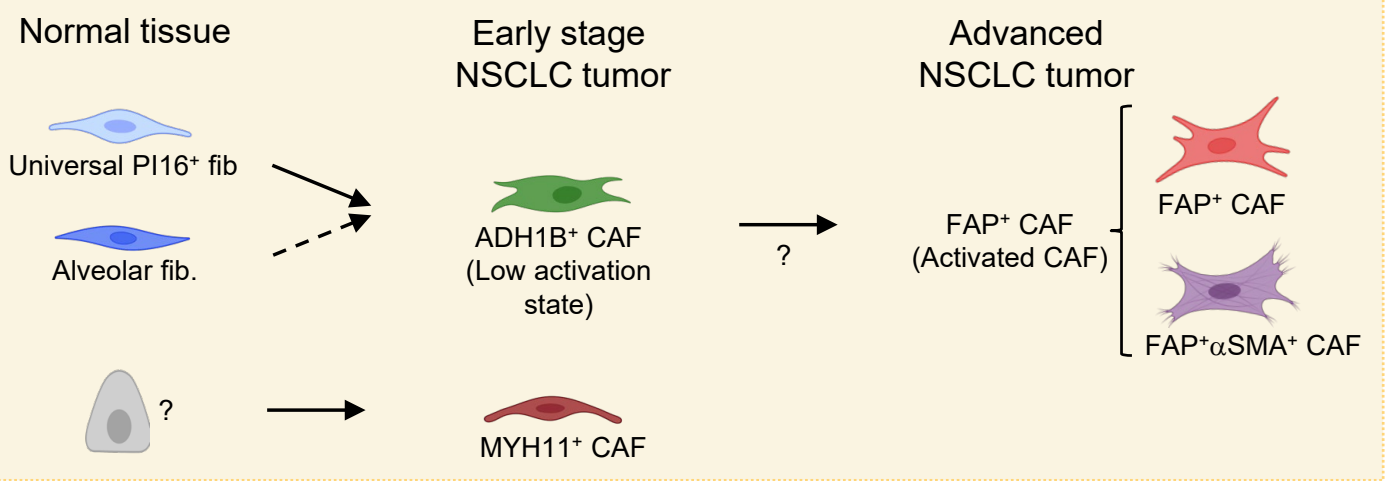


Fig. 7 | Working model. A, Graphical illustration of all stroma presentations found in this study. NSCLC samples enriched in $\mathrm{ADH}_{1} \mathrm{~B}^{+} \mathrm{CAF}$ throughout the stroma can be found with or without a single-cell layer of $\mathrm{MYH}_{1}{ }^{+} \alpha \mathrm{SMA}^{+} \mathrm{CAF}$ lining tumor cell aggregates. Those with MYH $11^{+} \alpha \mathrm{SMA}^{+} \mathrm{CAF}$ show increased $\mathrm{T}$ cell exclusion from the tumor nests. NSCLC samples enriched in $\mathrm{FAP}^{+} \mathrm{CAF}$ are found with variable abundance of $\mathrm{FAP}^{+} \alpha \mathrm{SMA}^{+} \mathrm{CAF}$. Stromal regions with high $\alpha$ SMA have reduced T cell accumulation, and tumor nests surrounded by several layers of $\mathrm{FAP}^{+} \alpha \mathrm{SMA}^{+} \mathrm{CAF}$ show a lower $\mathrm{T}$ cell infiltration. $\mathrm{B}$, Cartoon depicting the general distribution of fibroblast and CAF populations in adjacent lung tissue, early-stage NSCLC and advanced NSCLC, as well as the potential differentiation trajectories. 


\section{DISCUSSION}

318 The majority of patients fail to achieve clinical benefit using standard immune checkpoint

319 blockade, and as such, novel combination approaches are required to improve response (58).

320 Patients with T-cell excluded tumors have poor response to immune checkpoint blockade 321 compared to those with T-cell infiltrated tumors $(3,5,6)$ which raises the possibility that targeting

322 the mechanism of $\mathrm{T}$ cell exclusion would improve clinical responses. To this end, our study 323 provides a comprehensive map of the fibroblast compartment in human lung tumors at the single 324 cell level and with spatial resolution. We define the molecular and functional diversity of the 325 fibroblast compartment of lung tumors and determine how distinct CAF subsets may influence the 326 immune cell composition as well as T cell spatial organization.

328 Our analysis shows that the stroma in NSCLC lesions is dominated by either lowly activated $329 \mathrm{ADH}_{1 \mathrm{~B}}^{+} \mathrm{CAF}$, with or without $\mathrm{MYH} 11^{+} \alpha \mathrm{SMA}^{+} \mathrm{CAF}$, or highly activated $\mathrm{FAP}^{+} \mathrm{CAF}$, with 330 variable $\alpha$ SMA levels (Figure 7). $\mathrm{ADH}^{+} \mathrm{B}^{+} \mathrm{CAF}$ have higher activation levels than fibroblasts 331 found in the normal lung tissue, as seen by their expression profile and enrichment in the tumor 332 lesion, as well as by the clear spatial distinction between $\mathrm{CD}_{10}{ }^{+}$alv. fib. in adjacent tissue and $333 \mathrm{ADH} 1 \mathrm{~B}^{+} \mathrm{CD} 10^{\text {neg }} \mathrm{CAF}$ in the tumor as seen in multiplex $\mathrm{IHC}$. $\mathrm{FAP}^{+} \mathrm{CAF}$, by contrast, show 334 dramatic transcriptional differences from adjacent tissue fibroblasts, including high expression of 335 many previously established CAF markers like FAP, POSTN, and COL1A1 $(23,25)$. FAP ${ }^{+} \mathrm{CAF}^{-}$ 336 and $\mathrm{FAP}^{+} \alpha \mathrm{SMA}^{+} \mathrm{CAF}$ represent higher activation states compared to $\mathrm{ADH}^{+} \mathrm{B}^{+} \mathrm{CAF}$ and 337 occasionally form spatial gradients of $\mathrm{ADH} \mathrm{B}^{+}$-to-FAP $\mathrm{C}^{+} \mathrm{CAF}$, suggesting that $\mathrm{ADH} 1 \mathrm{~B}^{+} \mathrm{CAF}$ may 338 contribute to the $\mathrm{FAP}^{+} \mathrm{CAF}$ pool. Interestingly, $\mathrm{ADH}^{+} \mathrm{B}^{+} \mathrm{CAF}$ express transcriptional programs 339 of both alv. fib. and $P I 16^{+}$fib., suggesting that these two lung tissue cell types could give rise to 
$\mathrm{ADH}_{1 \mathrm{~B}^{+}} \mathrm{CAF}$. In vivo fate-mapping experiments will be needed to further investigate this

341 possibility.

343 We have also shown that $\mathrm{ADH}^{+} \mathrm{B}^{+} \mathrm{CAF}$ and $\mathrm{FAP}^{+} \mathrm{CAF}$ are associated with pathological and

344 histological subtypes, and tumor stage, $\mathrm{ADH}^{+} \mathrm{B}^{+} \mathrm{CAF}$ being associated with the adenocarcinoma

345 papillary subtype and stage 1 whereas $\mathrm{FAP}^{+} \mathrm{CAF}$ being enriched in the adenocarcinoma solid

346 subtype and squamous cell carcinomas, and in later stage. This association between CAF

347 populations and histological subtypes, which correlate with prognosis(43), may shed light on the

348 molecular programs behind the different NSCLC subtypes, and inform clinical trial inclusion

349 criteria when therapeutically targeting CAF subsets. Furthermore, our staining protocol for these

350 CAF subsets may help refine the categorization of histological subtypes. Beyond subtype and

351 stage, we found a significant association between $\mathrm{FAP}^{+} \mathrm{CAF}$ and the LCAM

352 inflammatory/activated immune phenotype (including SPP1+ Mo-Macs, IgG plasma cells, and

$353 \mathrm{PD}^{+} \mathrm{T}$ cells) which we previously described (29). This observation, in conjunction with the

354 distinct immunomodulatory profiles of $\mathrm{ADH}^{+} \mathrm{CAF}$ and $\mathrm{FAP}^{+} \mathrm{CAF}$, suggests that CAF

355 participate in shaping the immune response to the tumor.

357 Prior studies have suggested that activated CAF may play a role in $\mathrm{T}$ cell exclusion $(6,15,16)$.

358 Notably, $\mathrm{FAP}^{+} \mathrm{CAF}$ do not correlate with the $\mathrm{T}$ cell distribution pattern in NSCLC, an important

359 factor to keep in mind when developing therapeutics and may explain why strategies targeting

$360 \mathrm{FAP}^{+} \mathrm{CAF}$ have failed in human clinical trials so far $(18,59)$. In contrast, we have found two

361 distinct CAF populations with specific molecular programs and spatial organizations that

362 contribute to $\mathrm{T}$ cell exclusion. First, $\mathrm{FAP}^{+} \alpha \mathrm{SMA}^{+} \mathrm{CAF}$ are significantly correlated with regions 
363 of $\mathrm{T}$ cell exclusion in the tumor stroma and can form multiple layers at the tumor boundary and

364 restrict $\mathrm{T}$ cell contact with tumor cells. On the other hand, in a fraction of adenocarcinomas

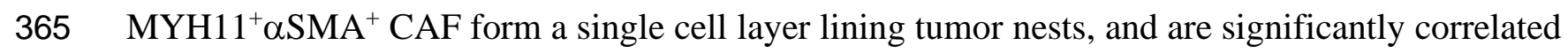

366 with immune cell exclusion from tumor regions, both within cancer lesions and across tumor

367 samples.

368

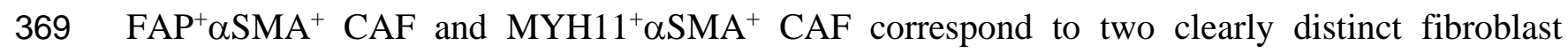

370 subsets, as observed through the scRNAseq data and in line with their presence in distinct tumors.

371 Notably, they display similarities, including high expression levels of ECM and contractility genes,

372 which may imply that they influence T cell spatial distribution through similar mechanisms, with

373 the production of fibers limiting T cell access to cancer cells. Supporting this, we previously

374 showed that ECM fiber orientation and density dictate the motility of T cells within the stroma and

375 restrict their interactions with tumor cells in human NSCLC (7). The profound differences in their

376 matrix programs (including type IV and IX collagens for $\mathrm{MYH} 11^{+} \alpha \mathrm{SMA}^{+} \mathrm{CAF}$ and type XI and

377 XII collagens for $\mathrm{FAP}^{+} \alpha \mathrm{SMA}^{+} \mathrm{CAF}$ ) also suggest that they may drive $\mathrm{T}$ cell marginalization by

378 forming different types of barrier to lymphocytes. While ECM degradation can improve T cell

379 infiltration $(7,54)$, targeting ECM molecules is challenging in patients given the low specificity

380 and the risk of on-target off-tumor toxicity. Here, our study paves the way to develop novel

381 strategies to differently target the two distinct cellular sources of these ECM molecules.

383 In summary, our study has identified several CAF populations that show greater heterogeneity

384 than previously established CAF classification, and provides novel therapeutic targets to pursue in

385 order to augment response to cancer immunotherapies. We demonstrate that pairing molecular and 
spatial analysis is crucial to understanding the true organization of the human TME and to

development of novel CAF targeting strategies for efficient anti-tumor combinations.

\section{SUPPLEMENTAL EXPERIMENTAL PROCEDURES}

\section{Human subjects}

In collaboration with the Biorepository and Department of Pathology tumor and adjacent non-involved lung samples were obtained from surgical specimens of patients undergoing resection at the Mount Sinai Medical Center (New York, NY). Informed consent was obtained in accordance with the following protocol reviewed and approved by the Institutional Review Board at the Icahn School of Medicine at Mount Sinai, IRB Human Subjects Electronic Research Applications 10-00472 and 10-00135.

\section{Tissue processing}

The non-involved lung and tumor tissue were weighed and cut into sections of 0.1-0.2 grams then placed into $5 \mathrm{~mL}$ microtube (Argos Technologies). Sections were minced with scissors and enzymatically digested in CO2-independent media (Fisher Scientific, 18045088) with Collagenase IV $0.25 \mathrm{mg} / \mathrm{ml}$ (Sigma-Aldrich, C5138-1G), Collagenase D $200 \mathrm{U} / \mathrm{ml}$ (Sigma-Aldrich, 11088882001), and DNAse $0.1 \mathrm{mg} / \mathrm{ml}$ (SigmaAldrich, DN25-1G) for 40 minutes at $37^{\circ} \mathrm{C}$ under $80 \mathrm{rpm}$ agitation. Cell suspensions were passed through a syringe with an 18-gauge needle 8-10 times, filtered through a 70 $\mu \mathrm{m}$ cell strainer, then lysed in red blood cell (RBC) buffer (Fisher Scientific, NC9067514). The cells were resuspended in buffer comprising of DPBS (Corning, D8537-6X500ML) with 5\% BSA (Equitech-Bio, BAH62-0500) and 1mM EDTA (Sigma-Aldrich, 46-034-Cl) then counted using hemocytometer and Trypan blue (Fisher Scientific, MT25900Cl).

\section{Flow cytometry sorting}

Cells were stained for EpCAM (Biolegend, clone 9C4), CD45 (Biolegend, clone HI30), CD29 (Biolegend, clone TS2/16), PDPN (Biolegend, clone NC-08), and LiveDead blue fluorescent dye (Thermo Scientific, L34963) for 30 minutes at $4^{\circ} \mathrm{C}$. Among live cells, EpCAM and CD45 were used to remove epithelial and immune cells respectively, while CD29, present on all stromal cells, was used to enrich for cells with intact surface markers (see fig. S1B). The $1.5 \mathrm{ml}$ collection tubes (Fisher Scientific, 05-408-129) were coated with $10 \%$ BSA to improve cell survival post sorting.

\section{Single cell RNA sequencing}

For each sample, up to an estimated 5,000 cells were loaded directly from the flow cytometry sort onto 10X Chromium chemistry kits. Kit versions for each sample are indicated in supplemental Table 1. Processing downstream of cell loading was performed by the Human Immune Monitoring Core at Icahn School of Medicine at Mount Sinai. Libraries were prepared according to manufacturer instructions and QC of the cDNA and final libraries was performed using CyberGreen qPCR library quantification assay. Sequencing was performed on Illumina sequencers to a depth of at least 80 million reads per library.

\section{Sequencing data analysis and unsupervised batch-aware clustering}

Transcriptomic library reads were aligned to the GRCh38 reference genome and quantified using Cell Ranger (v3.1.0).

Stromal cells isolated from tumor and adjacent lung samples were analyzed using an unsupervised batchaware clustering method we have recently described(30). First, stromal cells were filtered for cell barcodes recording $>800 \mathrm{UMI}$, with $<25 \%$ mitochondrial gene expression, and with less than defined thresholds of expression for genes associated with red blood cells, epithelial cells, macrophages, $T$ cells and plasma cells (Table 12). 13 tumor and 11 adjacent samples were clustered jointly. This EM-like algorithm iteratively updates both cluster assignments and sample-wise noise estimates until it converges, using a multinomial mixture model capturing the transcriptional profiles of the different cell-states and sample specific fractions 436 of background noise. We ran the algorithm described in Martin et al with minor modifications: Training and 
test set sizes per sample were 7500 and 2500 respectively. The best clustering initiation was selected from 1000 instead of $10000 \mathrm{k}$-means+ runs. For this clustering we included barcodes with more than $800 \mathrm{UMls}$ and used $K_{\text {reg_ds }}=0.2 ;\left(\mathrm{P}_{1}, \mathrm{P}_{2}\right)=\left(0^{\text {th }}, 50^{\text {th }}\right)$ percentiles; $K_{\text {reg }}=5 \cdot 10^{-6} ; \mathrm{k}=28$. Genes with high variability between patients across were not used in the clustering. Those genes consisted of mitochondrial, stress, metallothionein genes, immunoglobulin variable chain genes, HLA class I and II genes and 3 specific genes with variable/noisy expression: MALAT1, JCHAIN and XIST (Table 12). Ribosomal genes were excluded only from the k-means clustering (step 2.D as described in Martin et al.) (Table 12).

\section{Cell annotation}

Using the gene module analysis described earlier, we identified highly variable genes and explored their profiles reported in prior literature.

For stromal cell clusters, EC expressed multiple identifying markers like PECAM1, VWF, CLDN5, and $E M C N$ and lymphatics could be identified with TFF3, LYVE1, and PROX1. PVC were identified by combination of subset specific markers for and shared expression of contractile genes like ACTA2, TAGLN, MYL9 and TPM2. PvC subset specific genes included RGS5, COX4I2 and HIGD1B for pericytes and DES and ACTG2 for SM. Identifying fibroblast markers included those listed in the main text, PDGFRA, SPON1, and MMP2, but also DCN, FBLN1, LUM, COL1A2, RARRES2, CTGF.

A cluster (\#13) with contaminating epithelial cells was identified by the high expression of multiple keratin genes including KRT17 and KRT19. Contaminating macrophages were identified in cluster 6 by expression of CD45 (PTPRC), C1QB, C1QA, C1QC, and MARCO. Cluster 18 and 16 were excluded due to high mitochondrial gene content and hemoglobin genes, respectively. The annotation process for fibroblast subsets is described in the text.

\section{Histological staining}

Multiplexed IHC was performed according to the protocol developed by (28) with some modifications. Slides were baked at $37^{\circ} \mathrm{C}$ overnight, deparaffinized in xylene, then rehydrated. Antigen retrieval was done in citrate buffer ( $\mathrm{pH} 6$ or 9 ) (Dako, S2367 or 2369 ) at $95^{\circ} \mathrm{C}$ for 30 minutes, followed by incubation in $3 \%$ hydrogen peroxide for 15 minutes, then blocked using serum-free protein block solution (Dako, X0909) before adding primary antibody for 1 hour at room temperature or overnight at $4^{\circ} \mathrm{C}$. The primary antibody was detected using a secondary antibody conjugated to horseradish peroxidase followed by chromogenic revelation using 3-amino-9-ethylcarbazole (AEC) (Vector laboratories, SK4200). Slides were counterstained with hematoxylin (Sigma-Aldrich, HHS32-1L) and mounted with a glycerol-based mounting medium (Dako, C0563). Then the same slides were bleached and re-stained as previously described. Antibodies sources can be found in Supplemental Table 4. Masson's trichrome staining was performed by the Biorepository and Pathology core at the Icahn School of Medicine at Mount Sinai.

\section{Mass Cytometry by Time Of Flight (CyTOF)}

Samples were processed to a single cell suspension according to the tissue processing protocol listed earlier. Cell viability staining was achieved with Rh103 staining for 20 minutes at $37^{\circ} \mathrm{C}$ followed by staining with the CyTOF antibodies listed in Supplemental Table 4. Acquisition of the samples was performed by the HIMC at mount Sinai. All analysis of the CyTOF samples, including the creation of viSNE plots, was done using the Cytobank platform (https://www.cytobank.org/).

\section{ECM and immunomodulatory gene expression profiles}

Gene lists were sourced from (60) and (61) for ECM and immunomodulatory genes, respectively. Selected genes had to meet a mean expression threshold of 1 UMI per 2000 UMIs in $2 \%$ of cells in at least 1 cluster and meet a minimum 3-fold expression change between at least two clusters. Selected genes for display were further refined by qualitative analysis.

\section{Gene module analysis}

Gene correlation modules were generated using a similar method as previously described in (30). Briefly, cells are downsampled to 2000 total UMIs and highly variable genes are isolated. A gene-gene correlation matrix for the isolated gene set is computed for each sample over the cell population(s) of interest and 
correlation matrices are averaged following a Fisher Z-transformation. Applying the inverse transformation then results in the best-estimate correlation coefficients of gene-gene interactions across the dataset. Genes are clustered into modules using complete linkage hierarchical clustering over correlation distance. Ribosomal, mitochondrial, HLA and immunoglobulin genes were removed from the analysis prior to creation of gene modules as these genes were not of interest in this study, reflected patient genomic variability or were heavily influenced by contaminating plasma cells.

\section{Acquisition of TCGA dataset and histological subtypes}

The TCGA lung adenocarcinoma (LUAD) RNAseq data was downloaded using the GDCquery and GDCdownload functions from the TCGAbiolinks R package. GDCquery options included project="TCGALUAD", data.category="Transcriptome Profiling", data.type="Gene Expression Quantification", workflow.type="HTSeq - FPKM", experimental.strategy="RNA-Seq", and legacy=F. Whole exome sequencing data was downloaded using the GDCquery_Maf function with arguments tumor="TCGA-LUAD" and pipelines="mutect2". Clinical data was downloaded using the GDCquery_clinic function with arguments project="TCGA-LUAD" and type="clinical".

The dominant histological subtype for each TCGA tumor was sourced from (42).

\section{CAF gene signatures and LCAM in bulk analysis}

We performed gene module analysis to identify groups of co-expressed genes in the scRNAseq dataset as described above. To define cell type specific gene signatures, we first excluded genes expressed in nonfibroblast lineages, such as EC, PvC, epithelial, and immune cells. We then compared the gene module expression between fibroblast subsets. Due to their similarity, $\mathrm{FAP}^{+} \mathrm{CAF}$ and $\mathrm{FAP}^{+} \alpha \mathrm{SMA}^{+} \mathrm{CAF}$ were treated as one group and due to $A D H 1 B^{+} \mathrm{CAF}$ similarity to adjacent fibroblast clusters they were not contrasted with alv. fib. and $\mathrm{P} / 16^{+}$fib. Signatures were further refined by manually checking that expression was consistently enriched in the cell type of interest across at least 3 patients.

Bulk RNA gene expression values were log-transformed and z-scored. Cell type signatures, scored per sample, were calculated by taking the average of all genes within the signature. The derivation of LCAMhigh or LCAM ${ }^{\text {low }}$ is described in [Leader et al. 2021]. The cell types associated with each state were averaged to create an LCAM ${ }^{\text {high }}$ or LCAM ${ }^{\text {low }}$ score. The difference between LCAM ${ }^{\text {high }}$ and LCAM $^{\text {low }}$ was the final LCAM score. All signatures are calculated using only tumor samples, with sample ID ending in '-01A', and signatures were z-scored before graphing or other analysis. TCGA patients with their corresponding signatures scores can be found in Table 6.

\section{Histology analysis and overlays}

All histology analysis was performed using the open-source image analysis QuPath software (QuPath0.2.3, https://qupath.github.io/)(62) and ImageJ/Fiji(63,64).

To create overlayed images, scans exported from QuPath as OME.TIFF then imported into ImageJ using the BioFormats plugin(65). Alignment was done using the "Linear Stack Alignment with SIFT" plugin(66). The AEC and hematoxylin stains were extracted from individual scans using "colour deconvolution" and colored as desired.

Quantifying T cell infiltration and inter-patient CAF heterogeneity

Cropped scans were imported into a newly created project in QuPath and were aligned using the "interactive image alignment" plugin. Alignment information was saved using QuPath_script_1. Tumor and stroma regions were defined by applying the "create cytokine annotation" function on the keratin scans. The stroma and tumor annotations were transferred onto the aligned CD3, CD8, $\alpha$ SMA, ADH1B, and FAP scans with QuPath_script_2. On the CD3 and CD8 scans, positive cell detection was used to count the $\mathrm{CD}^{+}$and $\mathrm{CD}^{+}$cells. Distance to the tumor and stroma annotations was calculated using the "distance to annotation 2D" option and the measurements were exported as raw data to be analyzed in R. For figure 4E the stroma and tumor annotations were tiled using QuPath function "Create Tiles" and $\alpha$ SMA, ADH1B, and FAP scans the positively stained area were calculated using the QuPath training classifier.

$\underline{\text { Quantifying } T \text { cell infiltration and intra-patient CAF heterogeneity }}$ 
For the intra-patient analysis, we created a separate QuPath project with all the desired scans to analyze. The images were cropped and exported and then overlays were generated using an ImageJ script with the following steps:

551 a. Deconvolution (hematoxylin, AEC, residual)

552 b. Alignment on hematoxylin images

d. Threshold to remove background and then "Stack of Images"

The composite image was transferred back to QuPath for further analysis. Adjacent tissue regions on slides were excluded from the analysis and in the region to analyze we used the "train pixel classifier" to annotate Tumor nests versus Stroma; while $\alpha$ SMA, FAP and ADH1B areas were annotated using "QuPath train classifier". Dedicated script automated the tiling and quantification and resulted in 3 data files; cell information including staining intensity and distances to annotations, tile annotation parameters, and annotation measurements such as ADH1B stained area within an annotation. The resulting measurements were exported and analyzed in R.

\section{Quantifying MYH11+ $\alpha \mathrm{SMA}+\mathrm{CAF}$ boundary enrichment}

$\mathrm{MYH} 11^{+} \alpha \mathrm{SMA}+\mathrm{CAF}$ score (Figures 5C,5D) approximates the enrichment of these cells at the tumor nest boundary $(<10 \mu \mathrm{m})$ relative to their distal background density $(20-30 \mu \mathrm{m})$. Iterating over tiles, we counted the number of $\mathrm{MYH}_{11}{ }^{+} \alpha \mathrm{SMA}^{+}$double positive cells in each distance bin of $1 \mu \mathrm{m}$. The proximal value was defined as the quantile, 0.75 , of the number of cells in the bins within distance $<10 \mu \mathrm{m}$. The distal value was similarly defined as the quantile, 0.75 , of the number of cells in the bins with distances between $20-30 \mu \mathrm{m}$. The MYH $11^{+} \alpha \mathrm{SMA}^{+} \mathrm{CAF}$ score was defined as log2(proximal/distal). The 0.75 percentile was selected to maximize the sensitivity of detecting robust high-density regions.

573 Sequencing data will be available through GEO and accession number will be provided by 574 publication date. Code is available on our Github website: https://github.com/effiken/Grout_et_al.

\section{ACKNOWLEDGMENTS}

577 The authors thank the important contributions of patients who participated in this study. This

578 project was supported by Genentech, Inc. and carried out in collaboration with the Fondation ARC

579 pour la recherche sur le cancer. The computational work was supported by the Scientific

580 Computing at the Icahn School of Medicine at Mount Sinai and the Office of Research

581 Infrastructure of the National Institutes of Health under award number S10OD026880. The content

582 is solely the responsibility of the authors and does not necessarily represent the official views of

583 the National Institutes of Health. This manuscript was edited at Life Science Editors and the

584 cartoon illustrations were created using BioRender.com. We thank the Mount Sinai Flow 
585 Cytometry core, Human Immune Monitoring Center and the Biorepository and Pathology core for

586 their support. We thank Eliane Piaggio, Ana-Maria Lennon-Duménil, and Olivier Lantz for

587 carefully reading and commenting the manuscript.

588

589 AUTHOR CONTRIBUTIONS

590 HS, and EK conceived the project. JAG, FS, NT, and HS designed the experiments. JAG, EK, and

591 HS wrote the manuscript. JAG, PS, AML, EH, RP, SMü and EK performed computational

592 analysis. AW, MBB, and RF facilitated access to human samples. JAG, SMa, IP, EC, NT, MM,

593 SK, RV, LK, MP, MCA, AT and LW performed experiments and other analysis. AL conducted

594 patient consents and facilitated regulatory items. MM, and SJT provided key guidance. AML,

595 AHR, SG, JA, JCM, MBB, SMü, and TUM provided further intellectual input.

596

597 DECLARATION OF INTERESTS

598 Research support for this work was provided in part by Genentech, Inc. The authors declare no

599 other competing financial interests.

600

601 SUPPLEMENTAL TABLES

602 S1 - Patient clinical summary data

603 S2 - Patient cell counts per cluster

604 S3 - Adjacent and tumor sample percent composition of fibroblast clusters

605 S4 - Antibodies used in IHC, flow, and CyTOF

606 S5 - Positive staining area of ADH1B and FAP for IHC slides

607 S6 - TCGA patient expression of signatures 
S7 - CD8 and CD3 quantification across entire FFPE section

S8 - Slide tiling data for $\mathrm{FAP}^{+} \alpha \mathrm{SMA}^{+} \mathrm{CAF}$ - sample 24

610

S9 - Slide tiling data for $\mathrm{FAP}^{+} \alpha \mathrm{SMA}^{+} \mathrm{CAF}$ - sample 54

S10 - Slide tiling data for $\mathrm{MYH} 11^{+} \alpha \mathrm{SMA}^{+}-\mathrm{CAF}$ sample 13

S11 - Slide tiling data for MYH1 $1^{+} \alpha \mathrm{SMA}^{+}-\mathrm{CAF}$ sample 8

613 S12 - Gene lists used in scRNAseq clustering, in silico filtering and in figures

$614 \mathrm{~S} 13-\mathrm{CD}^{+} \mathrm{T}$ cell tumor density across NSCLC samples

\section{REFERENCES} and mortality worldwide: Sources, methods and major patterns in GLOBOCAN 2012. Int J

619 Cancer. 2015;136:E359-86.

620 2. Borghaei H, Paz-Ares L, Horn L, Spigel DR, Steins M, Ready NE, et al. Nivolumab versus 621 Docetaxel in Advanced Nonsquamous Non-Small-Cell Lung Cancer. New Engl J Medicine. 622 2015;373:1627-39.

623 3. Galon J, Bruni D. Approaches to treat immune hot, altered and cold tumours with combination 624 immunotherapies. Nat Rev Drug Discov. 2019;18:197-218.

625 4. Chen DS, Mellman I. Elements of cancer immunity and the cancer-immune set point. Nature. 626 2017;541:321-30.

627 5. Herbst RS, Soria J-C, Kowanetz M, Fine GD, Hamid O, Gordon MS, et al. Predictive 628 correlates of response to the anti-PD-L1 antibody MPDL3280A in cancer patients. Nature. $629 \quad 2014 ; 515: 563-7$.

630 6. Mariathasan S, Turley SJ, Nickles D, Castiglioni A, Yuen K, Wang Y, et al. TGF $\beta$ attenuates 631 tumour response to PD-L1 blockade by contributing to exclusion of T cells. Nature.

632 2018;554:544-8.

633 7. Salmon H, Franciszkiewicz K, Damotte D, Dieu-Nosjean M-C, Validire P, Trautmann A, et al. 634 Matrix architecture defines the preferential localization and migration of $\mathrm{T}$ cells into the stroma 635 of human lung tumors. J Clin Invest. 2012;122:899-910. 
8. Mueller SN, Germain RN. Stromal cell contributions to the homeostasis and functionality of the immune system. Nat Rev Immunol. 2009;9:618-29.

9. Bajénoff M, Egen JG, Koo LY, Laugier JP, Brau F, Glaichenhaus N, et al. Stromal Cell Networks Regulate Lymphocyte Entry, Migration, and Territoriality in Lymph Nodes. Immunity. 640 2006;25:989-1001.

641 10. Sixt M, Kanazawa N, Selg M, Samson T, Roos G, Reinhardt DP, et al. The Conduit System 642 Transports Soluble Antigens from the Afferent Lymph to Resident Dendritic Cells in the T Cell 643 Area of the Lymph Node. Immunity. 2005;22:19-29.

644 11. Kraman M, Bambrough PJ, Arnold JN, Roberts EW, Magiera L, Jones JO, et al. Suppression of Antitumor Immunity by Stromal Cells Expressing Fibroblast Activation Protein- $\alpha$. Science. 2010;330:827-30. Associated Fibroblasts. Front Immunol. 2018;9:414. reveals diverse stromal subsets associated with immune evasion in triple-negative breast cancer. Biorxiv. 2020;2020.06.04.135327. Facilitates Progression of Breast Cancer. Cell Reports. 2020;31:107701. Exclusion from Tumors. Cancer Res. 2020;80:1846-60. from FAP-expressing carcinoma-associated fibroblasts synergizes with anti-PD-L1 immunotherapy in pancreatic cancer. Proc National Acad Sci. 2013;110:20212-7. Antigen Targeting by a Humanised Monoclonal Antibody: An Early Phase II Trial of

666 Sibrotuzumab in Patients with Metastatic Colorectal Cancer. Oncol Res Treat. 2003;26:44-8.

667 19. Chen Y, McAndrews KM, Kalluri R. Clinical and therapeutic relevance of cancer-associated 668 fibroblasts. Nat Rev Clin Oncol. 2021;1-13. 
20. Öhlund D, Handly-Santana A, Biffi G, Elyada E, Almeida AS, Ponz-Sarvise M, et al. Distinct populations of inflammatory fibroblasts and myofibroblasts in pancreatic cancer. J Exp Medicine. 2017;214:579-96. Analysis Reveals Fibroblast Clusters Linked to Immunotherapy Resistance in Cancer. Cancer 674 Discov. 2020;10:1330-51. Associated Fibroblasts. Cancer Discov. 2019;9:1102-23.

23. Lambrechts D, Wauters E, Boeckx B, Aibar S, Nittner D, Burton O, et al. Phenotype molding of stromal cells in the lung tumor microenvironment. Nat Med. 2018;24:1277-89.

24. Chen Z, Zhou L, Liu L, Hou Y, Xiong M, Yang Y, et al. Single-cell RNA sequencing highlights the role of inflammatory cancer-associated fibroblasts in bladder urothelial carcinoma. 682 Nat Commun. 2020;11:5077. RNA Sequencing Reveals Stromal Evolution into LRRC15+ Myofibroblasts as a Determinant of Patient Response to Cancer Immunotherapy. Cancer Discov. 2020;10:232-53. cholangiocarcinoma growth by diverse cancer-associated fibroblast subpopulations. Cancer Cell. 2021;39:866-882.e11.

28. Remark R, Merghoub T, Grabe N, Litjens G, Damotte D, Wolchok JD, et al. In-depth tissue profiling using multiplexed immunohistochemical consecutive staining on single slide. Sci Immunol. 2016;1:aaf6925-aaf6925. stratification. Cancer Cell. 2021;39:1594-1609.e12. Anti-TNF Therapy. Cell. 2019;178:1493-1508.e20.

702 

Arteries and Veins As a Source of Mesenchymal Stem Cells. Stem Cells Dev. 2012;21:1299_ 705308. atlas of the human lung from single-cell RNA sequencing. Nature. 2020;587:619-25. associated fibroblasts and their markers. Int J Cancer. 2020;146:895-905. expression in rat and human mesothelioma. Cancer Res. 1994;54:3101-6.

714 37. Fletcher AL, Acton SE, Knoblich K. Lymph node fibroblastic reticular cells in health and 715 disease. Nat Rev Immunol. 2015;15:350-61.

716 38. Tang VW. Collagen, stiffness, and adhesion: the evolutionary basis of vertebrate 717 mechanobiology. Mol Biol Cell. 2020;31:1823-34.

718 39. Haruyama N, Sreenath TL, Suzuki S, Yao X, Wang Z, Wang Y, et al. Genetic evidence for 719 key roles of decorin and biglycan in dentin mineralization. Matrix Biol. 2009;28:129-36.

720 40. Park J, Ivey MJ, Deana Y, Riggsbee KL, Sörensen E, Schwabl V, et al. The Tcf21 lineage 721 constitutes the lung lipofibroblast population. Am J Physiol-lung C. 2019;316:L872-85.

722 41. Mecham RP. Elastin in lung development and disease pathogenesis. Matrix Biol. 2018;73:672320.

724 42. Collisson EA, Campbell JD, Brooks AN, Berger AH, Lee W, Chmielecki J, et al. 725 Comprehensive molecular profiling of lung adenocarcinoma. Nature. 2014;511:543-50.

726 43. Warth A, Muley T, Meister M, Stenzinger A, Thomas M, Schirmacher P, et al. The Novel 727 Histologic International Association for the Study of Lung Cancer/American Thoracic 728 Society/European Respiratory Society Classification System of Lung Adenocarcinoma Is a 729 Stage-Independent Predictor of Survival. J Clin Oncol. 2012;30:1438-46.

730 44. Fletcher AL, Turley SJ. Who am I? (re-)Defining fibroblast identity and immunological 731 function in the age of bioinformatics. Immunol Rev. 2021;302:5-9.

732 45. Chihara T, Suzu S, Hassan R, Chutiwitoonchai N, Hiyoshi M, Motoyoshi K, et al. IL-34 and 733 M-CSF share the receptor Fms but are not identical in biological activity and signal activation. 734 Cell Death Differ. 2010;17:1917-27. 
46. Menzies-Gow A, Ying S, Sabroe I, Stubbs VL, Soler D, Williams TJ, et al. Eotaxin (CCL11) and Eotaxin-2 (CCL24) Induce Recruitment of Eosinophils, Basophils, Neutrophils, and Macrophages As Well As Features of Early- and Late-Phase Allergic Reactions Following Cutaneous Injection in Human Atopic and Nonatopic Volunteers. J Immunol. 2002;169:2712-8.

47. Schall TJ, Bacon K, Toy KJ, Goeddel DV. Selective attraction of monocytes and T lymphocytes of the memory phenotype by cytokine RANTES. Nature. 1990;347:669-71. CD4+ and CD8+ T cells in response to MIP-1 alpha and MIP-1 beta. Science. 1993;260:355-8.

743 49. Castellino F, Huang AY, Altan-Bonnet G, Stoll S, Scheinecker C, Germain RN. Chemokines enhance immunity by guiding naive CD8+ T cells to sites of CD4+ T cell-dendritic cell interaction. Nature. 2006;440:890-5. Epithelial Cell Cytokine that Regulates T Cell Differentiation by Conditioning Dendritic Cell Maturation. Annu Rev Immunol. 2007;25:193-219.

51. Hegde PS, Karanikas V, Evers S. The Where, the When, and the How of Immune Monitoring for Cancer Immunotherapies in the Era of Checkpoint Inhibition. Clin Cancer Res. 2016;22:1865-74.

52. Tauriello DVF, Palomo-Ponce S, Stork D, Berenguer-Llergo A, Badia-Ramentol J, Iglesias $\mathrm{M}$, et al. TGF $\beta$ drives immune evasion in genetically reconstituted colon cancer metastasis.

754 Nature. 2018;554:538-43. extracellular matrix genes link cancer-associated fibroblasts to immune evasion and immunotherapy failure. Nat Commun. 2018;9:4692. infiltration and antitumor activity of CAR-redirected T lymphocytes. Nat Med. 2015;21:524-9.

55. Vázquez-Villa F, García-Ocaña M, Galván JA, García-Martínez J, García-Pravia C, Menéndez-Rodríguez P, et al. COL11A1/(pro)collagen 11A1 expression is a remarkable biomarker of human invasive carcinoma-associated stromal cells and carcinoma progression. Tumor Biol. 2015;36:2213-22. 
58. Herbst RS, Baas P, Kim D-W, Felip E, Pérez-Gracia JL, Han J-Y, et al. Pembrolizumab versus docetaxel for previously treated, PD-L1-positive, advanced non-small-cell lung cancer (KEYNOTE-010): a randomised controlled trial. Lancet. 2016;387:1540-50.

771 59. Sahai E, Astsaturov I, Cukierman E, DeNardo DG, Egeblad M, Evans RM, et al. A 772 framework for advancing our understanding of cancer-associated fibroblasts. Nat Rev Cancer. $773 \quad 2020 ; 20: 174-86$.

774 60. Naba A, Clauser KR, Lamar JM, Carr SA, Hynes RO. Extracellular matrix signatures of 775 human mammary carcinoma identify novel metastasis promoters. Elife. 2014;3:e01308.

776 61. Ramilowski JA, Goldberg T, Harshbarger J, Kloppmann E, Kloppman E, Lizio M, et al. A 777 draft network of ligand-receptor-mediated multicellular signalling in human. Nat Commun. $778 \quad 2015 ; 6: 7866$.

779 62. Bankhead P, Loughrey MB, Fernández JA, Dombrowski Y, McArt DG, Dunne PD, et al. 780 QuPath: Open source software for digital pathology image analysis. Sci Rep-uk. 2017;7:16878.

781 63. Schindelin J, Arganda-Carreras I, Frise E, Kaynig V, Longair M, Pietzsch T, et al. Fiji: an 782 open-source platform for biological-image analysis. Nat Methods. 2012;9:676-82.

783 64. Schneider CA, Rasband WS, Eliceiri KW. NIH Image to ImageJ: 25 years of image analysis. $784 \quad$ Nat Methods. 2012;9:671-5.

785 65. Linkert M, Rueden CT, Allan C, Burel J-M, Moore W, Patterson A, et al. Metadata matters: 786 access to image data in the real world. J Cell Biology. 2010;189:777-82.

787 66. Lowe DG. Distinctive Image Features from Scale-Invariant Keypoints. Int J Comput Vision. $788 \quad 2004 ; 60: 91-110$. 